ARTICLE

\title{
The secreted endoribonuclease ENDU-2 from the soma protects germline immortality in C. elegans
}

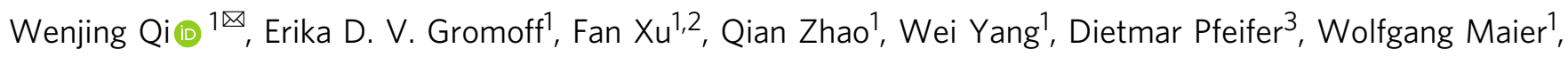
Lijiang Long $^{1} \&$ Ralf Baumeister ${ }^{1,2,4,5}$

Multicellular organisms coordinate tissue specific responses to environmental information via both cell-autonomous and non-autonomous mechanisms. In addition to secreted ligands, recent reports implicated release of small RNAs in regulating gene expression across tissue boundaries. Here, we show that the conserved poly-U specific endoribonuclease ENDU-2 in C. elegans is secreted from the soma and taken-up by the germline to ensure germline immortality at elevated temperature. ENDU-2 binds to mature mRNAs and negatively regulates mRNA abundance both in the soma and the germline. While ENDU-2 promotes RNA decay in the soma directly via its endoribonuclease activity, ENDU-2 prevents misexpression of soma-specific genes in the germline and preserves germline immortality independent of its RNA-cleavage activity. In summary, our results suggest that the secreted RNase ENDU-2 regulates gene expression across tissue boundaries in response to temperature alterations and contributes to maintenance of stem cell immortality, probably via retaining a stem cell specific program of gene expression.

\footnotetext{
${ }^{1}$ Bioinformatics and Molecular Genetics (Faculty of Biology), Albert-Ludwigs-University Freiburg, Freiburg, Germany. ${ }^{2}$ Spemann Graduate School of Biology and Medicine (SGBM), Albert-Ludwigs-University Freiburg, Freiburg, Germany. ${ }^{3}$ Department of Hematology, Oncology and Stem Cell Transplantation, Medical Center-University of Freiburg, Faculty of Medicine, University of Freiburg, Freiburg, Germany. ${ }^{4}$ Center for Biochemistry and Molecular Cell Research (ZBMZ, Faculty of Medicine), Albert-Ludwigs-University Freiburg, Freiburg, Germany. ${ }^{5}$ Signalling Research Centers BIOSS and CIBSS, Albert-LudwigsUniversity Freiburg, Freiburg, Germany. ${ }^{凶}$ email: wenjing.qi@biologie.uni-freiburg.de
} 
G erm cells are the only type of cells within an organism that deliver genetic and epigenetic materials to the offspring. Germ cells are therefore distinct from the somatic cells in their ability to maintain totipotency for production of an entire organism upon fertilization, and their immortality to allow reproduction for unlimited future generations. In C. elegans, loss of germline immortality typically results in steadily increased incidence of sterility of initially fertile animals over generations. To date, telomerase-mediated telomere maintenance, balanced heterochromatic H3K9 methylation, and euchromatic H3K4/ H3K36 methylation are the prevalent known molecular mechanisms involved in maintenance of germline immortality ${ }^{1-4}$. Piwi RNA mutants and mutations in multiple components of a nuclear RNA interference (RNAi) pathway that promotes the inheritance of germline RNAi also result in a temperaturedependent mortal germline (Mrt) phenotype ${ }^{5,6}$. Failure to maintain germline immortality in Piwi RNA mutants has recently been explained by transgenerational silencing of histone genes ${ }^{7}$. These studies, however, focused almost exclusively on autonomous mechanisms within the germline. It is unknown whether somatic signaling may contribute to maintenance of germline immortality.

C. elegans ENDU-2 belongs to a conserved but less studied family of proteins containing proposed poly- $\mathrm{U}$ specific endoribonuclease (XendoU) domains. While human EndoU (PP11 placental protein 11) is used as a cancer marker gene, Viral EndoU Nsp15 is highly conserved in all known coronaviruses and has been suggested to promote viral RNA replication and limit innate immunity response of the host cells ${ }^{8,9}$. C. elegans ENDU-2 has been shown to regulate cold stress response ${ }^{10}$. Very recently, ENDU-2 was reported to regulate nucleotides metabolism and germline proliferation in response to alterations in nucleotide levels and genotoxic stresses ${ }^{11}$.

Here we report that secretion of the poly- $U$ specific endoribonuclease ENDU-2 from the soma to the germline preserves germline immortality at elevated temperature. We find that ENDU-2 binds to mature mRNAs and downregulates mRNA levels both in the soma and in the germline. In addition, RNAbinding and -cleavage are two separable activities utilized by ENDU-2 to control gene expression via distinct mechanisms. In the soma, ENDU-2 relies on its endoribonuclease activity to negatively regulate a subset of its mRNA targets. In the germline, ENDU-2 prevents misexpression of soma-specific genes and ensures stem cell immortality primarily via its RNA-binding activity, suggesting an essential role of ENDU-2 in retaining the stem cell-specific program of gene expression. In summary, our data suggest that the soma sends ENDU-2 as a messenger to the germline to control gene expression across tissue boundaries in response to temperature alterations.

\section{Results}

Loss of $e n d u-2$ causes a temperature-dependent Mrt phenotype. Freshly outcrossed $e n d u-2(l f)$ mutants were phenotypically similar to wild type animals in the first few generations, except displaying slightly reduced germline proliferation, an egg-laying defect (Egl) due to abnormal development of the vulva, and a moderate reduction in adult lifespan at $20^{\circ} \mathrm{C}$ (Supplementary Fig. 1). Longterm strain maintenance at $20^{\circ} \mathrm{C}$ was difficult, since the endu-2(lf) mutants showed gradually increased sterility that, however, could be reset by additional outcrosses with wild type animals. We examined the fertility of two endu-2 alleles tm 4977 and by188 over generations after four additional outcrosses. tm 4977 allele has a $620 \mathrm{bp}$ deletion starting from the $5^{\prime}$ UTR to the end of the third intron, while the $20 \mathrm{bp}$ deletion in by188 causes an early stop codon in the first exon. Therefore, these two alleles are probably null mutants. Both tm4977 and by188 alleles became sterile at $20^{\circ} \mathrm{C}$ after about 15-20 generations (Fig. 1a, Supplementary Fig. 2a). Since we did not observe a significant reduction of the number of germ nuclei in the mitotic zone across generations, sterility was not the consequence of declining germline proliferation (Supplementary Fig. 2b). This result also indicates that the Mrt phenotype is probably not caused by altered activity of CTPS-1, an ENDU-2 controlled cytidine triphosphate synthase playing an essential role in germline proliferation ${ }^{11}$. Instead, endu2 day 1 adult animals in the generations with highly penetrant sterile phenotype showed pleiotropic defects in the germline. The most prominent defects were abnormal cell death $(38 \%, n=105)$ and increased number of apoptotic corpses ( $31 \%$ with $\geq 2$ corpses per gonad arm, $n=105$ ) (Fig. 1b). We also observed prolonged spermatogenesis $24 \mathrm{~h}$ after mid-L4 stage $(11 \%, n=105)$, whereas wild type animals had completely switched spermatogenesis to oogenesis. Furthermore, endomitosis occurred in the mitotic region $(14 \%, n=25)$ (Fig. 1b). In the generations displaying strong sterile phenotype, endu-2(lf) additionally showed high incidence of male progeny (Him, $14 \%, n=218$ ) and increased occurrence of other phenotypes (Rol, Dpy, Sma phenotypes, in total $10 \%, n=218$ ). The latter were probably caused by elevated somatic mutation rate, since these phenotypes were not heritable. The sterile phenotype was $100 \%$ penetrant at $25^{\circ} \mathrm{C}$ already at generation 6-10, but could not be detected at $15^{\circ} \mathrm{C}$ (Supplementary Fig. $2 \mathrm{c}$ and d). Notably, the highly penetrate sterility could be fully reversed within several generations by transferring the animals to $15^{\circ} \mathrm{C}$ (Fig. 1a). Taken together, these results suggest an essential role of ENDU- 2 in preserving germline immortality at elevated temperature and the existence of additional mechanisms to compensate for the loss of endu- 2 at $15^{\circ} \mathrm{C}$.

ENDU-2 is a secreted protein. A previous report has suggested a wide-spread expression of endu-2 in somatic tissues ${ }^{10}$, but did not report ENDU-2 localization in the germline. To retest the expression pattern of ENDU-2, we generated several independent transcriptional and translational endu-2 reporters with EGFP as well as a CRISPR/Cas9 EGFP knock-in strain at the endogenous endu-2 locus (expression constructs are shown in Supplementary Fig. 3a). A transcriptional fusion reporter gene, harboring $4 \mathrm{~kb}$ of endu-2 upstream sequences, was expressed only in the intestine (Supplementary Fig. 3b). A translational reporter harboring the same upstream sequences and the entire genomic region of endu2, fused to EGFP, rescued Egl, reduced germline proliferation, and short lifespan phenotypes (Supplementary Fig. 1a, $\mathrm{c}$ and d). The CRISPR/Cas9 EGFP knock-in strain did not display the Mrt phenotype (Supplementary Fig. 1e). These results suggest that ENDU-2::EGFP fusion proteins in the transgenic and the EGFP knock-in strains are functional. We observed ENDU-2::EGFP protein in the cytoplasm of intestine, somatic gonad, and coelomocytes in both endu-2::EGFP transgenic and the CRISPR/Cas9 EGFP knock-in strains (Fig. 2a and Supplementary Fig. 3c). Unlike suggested in the study of Ujisawa et al., we did not detect ENDU-2::EGFP in the musculature or in the neurons. Instead, we noticed extracellularly localized ENDU-2::EGFP, such as in the interspace between uterine wall and embryos (Fig. 2a and Supplementary Fig. 3c), suggesting that ENDU-2 may be a secreted protein. ENDU-2 indeed contains a predicted N-terminal (1-19 amino acid) secretion signal peptide for endoplasmic reticulum (ER) targeting, indicating that the protein is destined toward the secretory pathway. Fusion of this secretion signal peptide to the $\mathrm{N}$-terminus of a EGFP reporter gene expressed exclusively in the neurons led to only weak expression level of EGFP protein in the neurons, but an EGFP signal in the extracellular space and the coelomocytes, cells that are known to endocytose secreted 
a

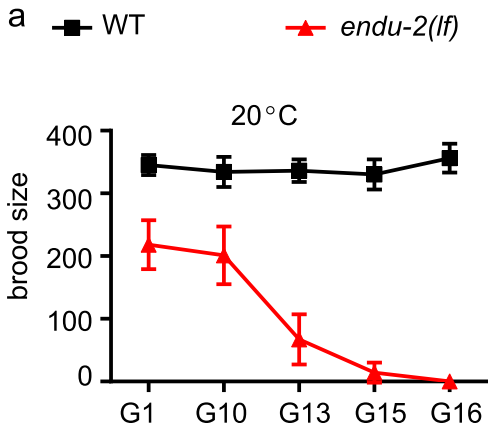

$15^{\circ} \mathrm{C}$

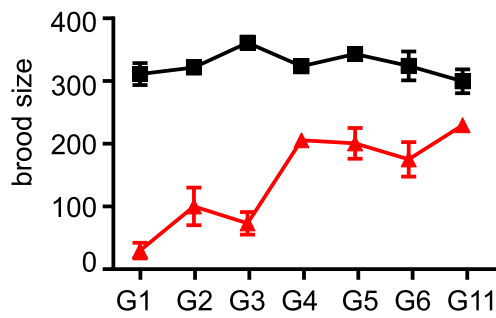

b
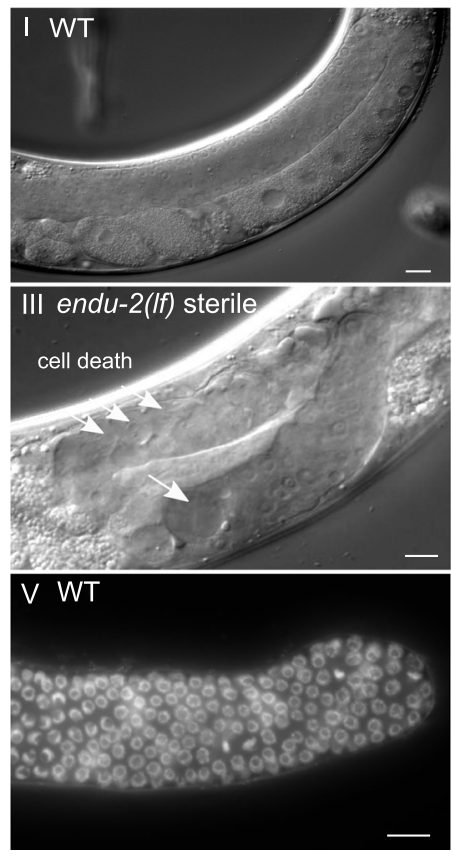
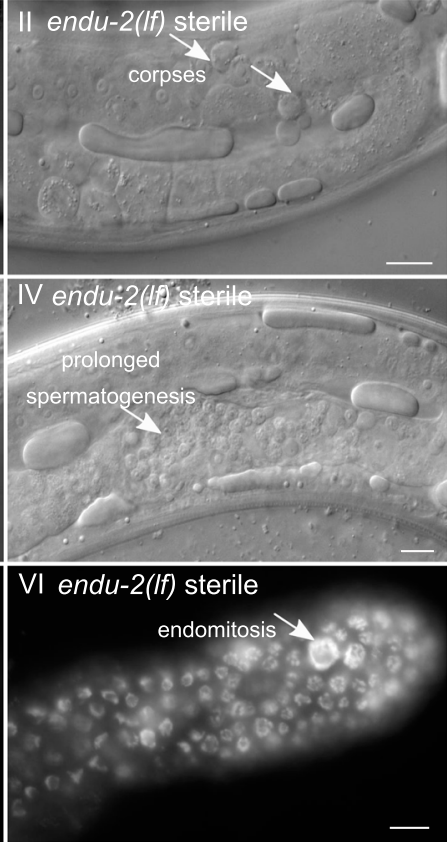

Fig. 1 endu-2 mutant shows a temperature-dependent Mrt phenotype. a Upper graph: Brood size of endu-2(tm4977), after 4× initial backcrosses with wild type (GO), decreases over generations at $20^{\circ} \mathrm{C}$. Lower graph: The reproduction defect of endu-2(tm4977) animals is reversible by shifting the animals to $15^{\circ} \mathrm{C}$. To obtain this graph, adult animals from $\mathrm{G} 6$ that had been maintained at $25^{\circ} \mathrm{C}$ were shifted back to $15^{\circ} \mathrm{C}$ and counted as $\mathrm{G} 0$. As the numbers of generation to reach $100 \%$ sterility at $20^{\circ} \mathrm{C}$ varied from 10 to 20 among the four biological replicates, only one represented replicate with $n=15$ animals for each generation is shown. The data are mean \pm SEM. b The sterile endu-2(tm4977) animals display multiple defects in germline mitosis and meiosis. (I-IV) DIC images of wild type and sterile endu-2(tm4977) animals $24 \mathrm{~h}$ after mid-L4 stage. The white arrows point to (II) increased number of germline apoptotic corpses; (III) empty gonad due to germ cell death; (IV) prolonged spermatogenesis; (V) DAPI staining of germline proliferating zone of day-one wild type and (VI) sterile endu-2(tm4977) adults, respectively. White arrows in $\mathrm{VI}$ point to abnormally large chromosomes due to endomitosis. Scale bar $10 \mu \mathrm{m}$. $\mathrm{N}=$ 3 biological replicates.

proteins from the pseudocoelic fluid (Fig. 2b). In addition, removing the $\mathrm{N}$-terminal secretion signal peptide from ENDU-2 $\left(\Delta_{\mathrm{ss}}\right.$ ENDU-2::EGFP) resulted in strong localization of $\Delta_{\mathrm{ss}}$ ENDU$2::$ EGFP only in the intestine but not in the coelomocytes (Supplementary Figs. $3 e$ and $4 a$ ). These results together suggest that the secretion signal peptide composed of the first 19 amino acids of ENDU-2 is necessary and sufficient to trigger secretion of a protein. Strikingly, we noticed that another transgenic reporter expressing 3xFlag::ENDU-2::EGFP was expressed strongly in the intestinal and also weakly in some head neurons, muscle cells in the head region, and anal depressor muscle cells, corroborating the report from Ujisawa et al. (Supplementary Fig. 3d). We speculate that the N-terminal 3xFlag fusion may prevent secretion by impairing binding of the secretion signal peptide by signal recognition particle (SRP), thus allowing detection of the weak expression in the muscles and neurons. In addition, expression in the head neurons and muscle cells might possibly be controlled by promoter signals localized in the first intron since this was the only sequence absent in the transgene expressing $\Delta_{s s} e n d u-2::$ EGFP (Supplementary Fig. 3a), in which we failed to observe muscular or neuronal localization. Moreover, when we expressed endu-2::EGFP selectively either in the neurons (unc-119 promoter), muscles (myo-3 promoter) or intestine ( $v$ ha- 6 promoter), ENDU-2::EGFP was always detected in the coelomocytes (Supplementary Fig. 4a), indicative for its secretion from these tissues. Notably, expressing endu-2::EGFP specifically in either neurons or muscles of heat-stressed animals resulted in ENDU-2::EGFP localization in the pharynx (Supplementary Fig. 4b), suggesting that either secretion or uptake of ENDU-2 in these tissues could be modulated by temperature.
Secretion of ENDU-2 from the soma to the gonad protects germline immortality. It is generally accepted that, in C. elegans, multi-copy extrachromosomal arrays are silenced and not expressed in the germline ${ }^{12}$. However, we found that extrachromosomal endu-2::EGFP transgenes rescued the Mrt germline phenotype of endu-2(lf) animals (Fig. 3a), suggesting that somatic ENDU-2 might preserve germline immortality across tissue boundaries. Our hypothesis was that secreted ENDU-2 could be endocytosed by the gonad. To test this assumption, we first asked whether ENDU-2 protein could be detected in the germline. All of our endu-2::EGFP transgenic reporters displayed weak expression levels unless secretion was blocked. Therefore, we could only occasionally observe faint ENDU-2::EGFP in few oocytes (Supplementary Fig. 4c). Via GFP-antibody staining we detected ENDU-2::EGFP in punctate structures in the germline at 15,20 , and $25^{\circ} \mathrm{C}$ (Fig. 2c and Supplementary Fig. 5). In contrast, $\Delta_{\text {ss }}$ ENDU-2::EGFP was not detected in the germline despite of its high expression level (Fig. $2 c$ and Supplementary Fig. 3F). In addition, fusing the secretion signal peptide of another secreted protein SEL- $1^{13}$ to $\Delta_{\mathrm{ss}}$ ENDU-2::EGFP reactivated secretion and its localization in the germline (Fig. $2 \mathrm{c}$ and Supplementary Fig. 3e). These observations indicate that secretion of ENDU-2 is indispensable for its gonadal localization. Moreover, we performed single molecular FISH (smFISH) staining to visualize endu-2 mRNA. endu-2 mRNA signals were visible predominantly in the intestine, but were clearly absent from the germline (Fig. 2d). Furthermore, sequencing of RNA extracted from isolated gonads also failed to detect endu-2 mRNA in the wild type gonad (Supplementary Data 3). Taken together, these results implicate that somatically expressed ENDU-2 protein is secreted and can be taken up by the gonad. 


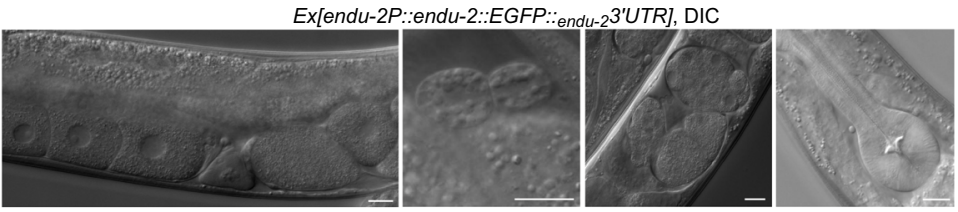

Ex[endu-2P::endu-2::EGFP::endu-2 $\left.3^{\prime} U T R\right]$, GFP

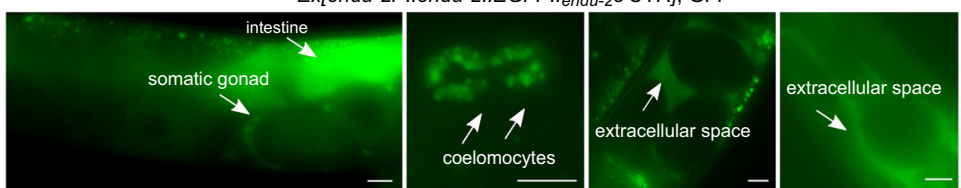

coelomocytes
C

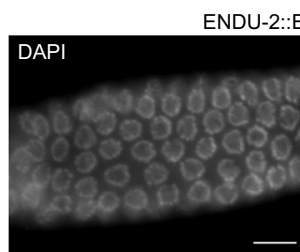

$\Delta_{\mathrm{ss}} \mathrm{ENDU}-2::$

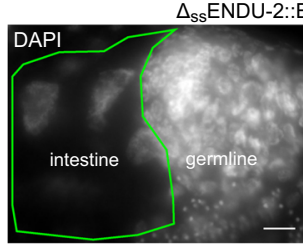

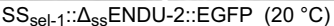

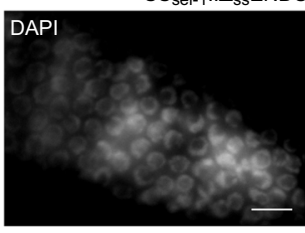

d

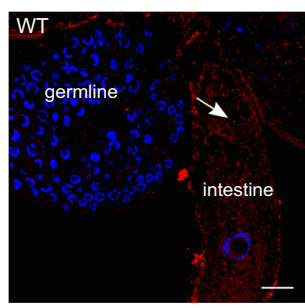

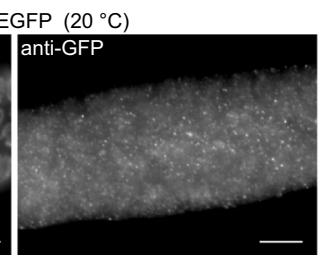

EGFP $\left(20^{\circ} \mathrm{C}\right)$
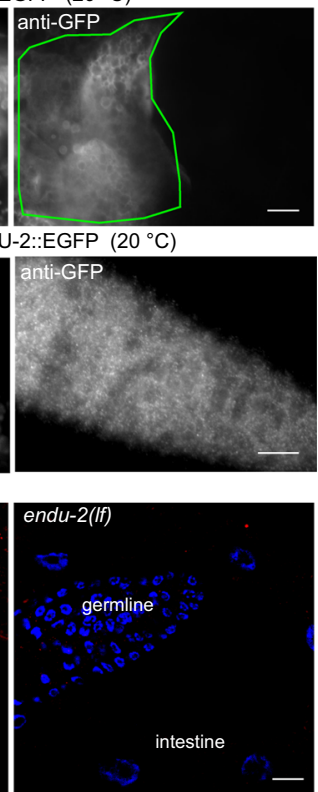

b

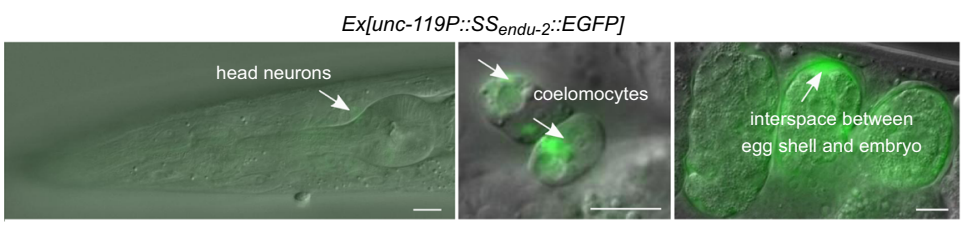

Ex[unc-119P::EGFP]
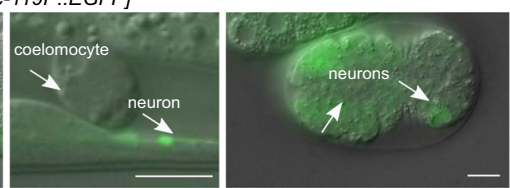

Fig. 2 ENDU-2 is a secreted protein. a Fluorescence micrographs of transgenic endu-2(tm4977); byEx1814[endu-2P::endu-2::EGFP:: endu-2 $\left.3^{\prime} U T R\right]$ animals. ENDU-2::EGFP is detected in the intestine, the somatic gonad, coelomocytes, and extracellular space between the uterus and embryos. $N=5$ independent experiments. b Fusion of the 1-19 amino acids of ENDU-2 (SS endu-2 $_{2}$ ) to neuronal-specific expressed EGFP is sufficient for secretion of EGFP protein. Upper panels show localization of unc-119P::EGFP which is detected only in neuronal cells (strong) of animals and embryos. Lower panels are images showing localization of unc-119P::SS endu-2::EGFP which is detected in the neurons (weak), coelomocytes and interspace between egg shell and embryos, indicative of efficient secretion of EGFP mediated by the secretion signal peptide of ENDU-2. Images are representative for more than 20 animals, analyzed by using a $\times 40$ objective. c Fluorescence micrographs of GFP-antibody staining of endu-2(tm4977); byEx1375[endu-2P::endu-2::EGFP], endu-2(tm4977); byEx1449[endu-

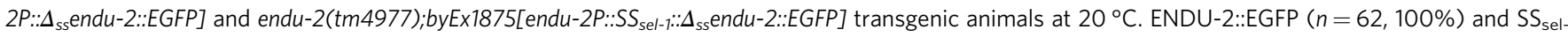
$\because: \Delta_{\text {ss }}$ ENDU-2::EGFP $(n=21,100 \%)$ but not $\Delta_{\text {ss }}$ ENDU-2::EGFP $(n=26,0 \%)$ is detected in the germline. $N=2$ independent replicates. $\mathbf{d}$ smFISH staining reveals presence of endu-2 mRNA in the intestine (white arrow), but not in the germline. Blue: DAPI stained nuclear DNA. Red: smFISH probes stained mRNA. $N=4$. Scale bar $10 \mu \mathrm{m}$ for all images in this Figure.

Next, we asked from which tissue ENDU-2 ensures germline immortality. Expressing ENDU-2 specifically in the intestine or the neurons, but not the muscle or somatic gonad, was sufficient to rescue the Mrt phenotype, indicating a non-cell-autonomous ENDU-2 signal from the neurons and intestine to the germline (Fig. 3b). In addition, expressing the secretion-deficient $\Delta_{s s}$ ENDU-2::EGFP failed to rescue the Mrt phenotype (Fig. 3c). Furthermore, $S_{\text {sel-1 }}: \Delta_{\text {ss }}$ ENDU-2::EGFP rescued the Mrt phenotype (Fig. 3c), suggesting that guiding ENDU-2 into ER-Golgi secretory pathway via a canonical secretion signal suffices for functions of ENDU-2 in the germline.

To directly test whether loss of somatic endu-2 expression is sufficient to induce a mortal germline, we performed endu-2 RNAi knock-down in a $p p w-1$ mutant background in which germline RNAi does not function ${ }^{14}$. Although neither wild type nor $p p w-1$ mutants upon endu-2 RNAi displayed a fully penetrant Mrt phenotype within 15 generation, the soma-specific endu-2 RNAi resulted in gradually reduced brood sizes over generations in both $p p w-1$ and wild type background, indicating that somatically expressed endu-2 mRNA is required for normal reproduction (Supplementary Fig. 6a). The failure to obtain a fully penetrant Mrt phenotype in this experiment may be due to the relative inefficacy of RNAi in the nervous system, a tissue that expresses endu-2 to ensure germline immortality.

ENDU-2 affects the oocytes to preserve germline immortality independent of the nuclear RNAi pathway. To know whether ENDU-2 affects oocytes or sperm to ensure germline immortality, we tested the parental contribution of ENDU-2 during sexual reproduction. For this purpose, we crossed endu-2(+/+) wild type parents with either males or hermaphrodites of endu-2(-/-) that had been grown at $25^{\circ} \mathrm{C}$ for five to seven generations and displayed strongly reduced brood size and high percentage of sterility (Fig. 3d). Whereas the heterozygous progeny of endu- $2(+/+)$ mothers had brood sizes with typically more than $100 \mathrm{~F} 2$ animals, the brood size of F1 cross-progeny derived from endu-2(-/-) mothers was as low as that of their mothers. We conclude that ENDU-2 function is required predominantly in the oocytes to preserve germline immortality.

The temperature-dependent Mrt phenotype of endu-2 mutants resembles that of hrde-1 mutants in the nuclear RNAi pathway ${ }^{5,15}$. In addition to loss of germline immortality, hrde-1 is also defective in multigenerational inheritance of germline 


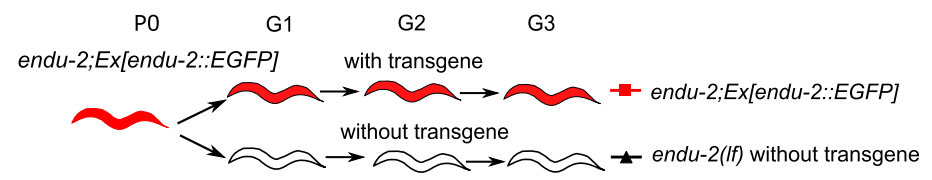

endu-2;Ex[endu-2P::endu-2::EGFP]

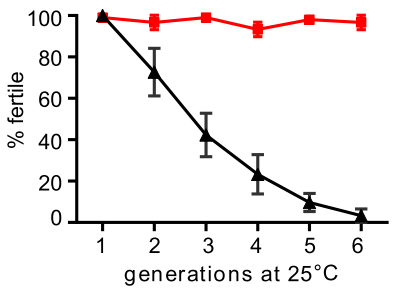

b
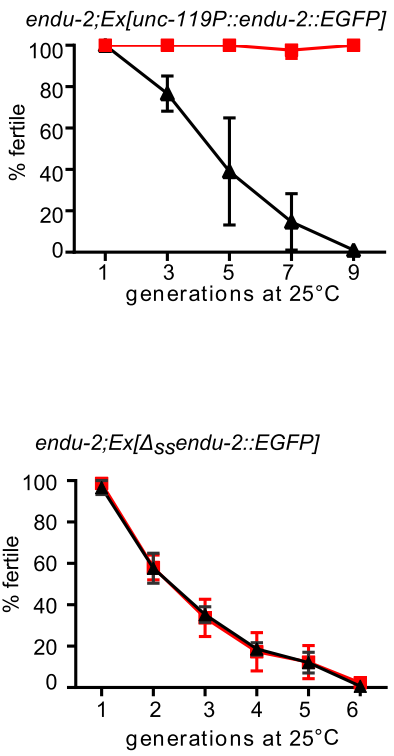

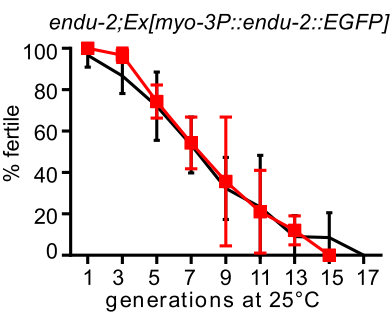
generations at $25^{\circ} \mathrm{C}$

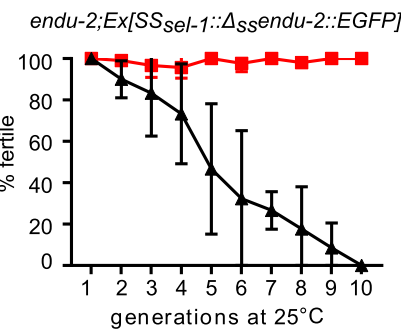

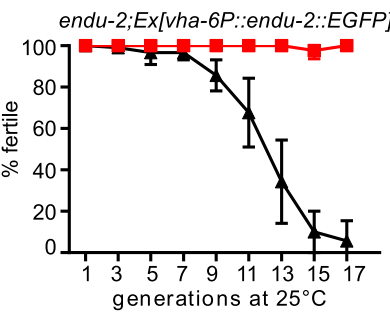

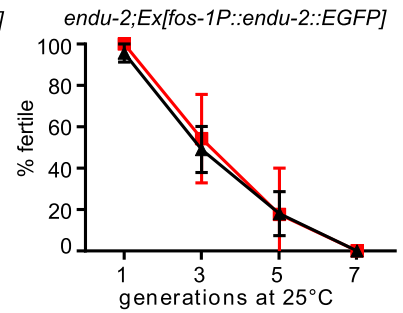

d

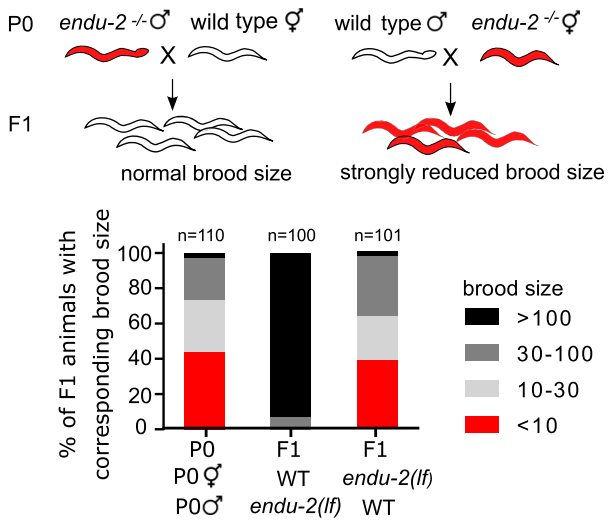

Fig. 3 Maternally provided ENDU-2 from the soma contributes to maintenance of germline immortality. a endu-2(wt)::EGFP rescues the Mrt phenotype at $25^{\circ} \mathrm{C}$. Left panel shows the experimental strategy for all rescue experiments with endu-2 transgenes in this study. endu-2(-/-) daughter generation (G1) that has lost the extrachromosomal rescue constructs expressing endu-2::EGFP was isolated. This and the following endu-2(-/-) generations (G2 to $\mathrm{Gn}$ ) were compared to endu-2;Ex[endu-2:.EGFP] derived from the same PO animal. Data are mean $\pm \mathrm{SD}, N=3$. $\mathbf{b}$ Expression of endu-2(wt)::EGFP from

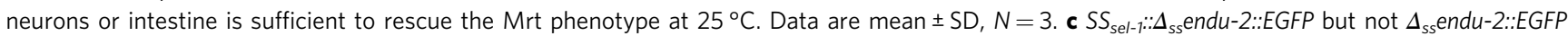
transgene rescues the Mrt phenotype at $25^{\circ} \mathrm{C}$. Data are mean $\pm \mathrm{SD}, \mathrm{N}=3$. d ENDU-2 primarily affects oocyte to maintain germline immortality. Data are pooled data from three biological replicates with similar tendencies in results.

RNAi. To test whether ENDU-2 acts in the nuclear RNAi pathway, we examined oma-1 RNAi inheritance that suppresses embryonic lethality of an oma-1 gain-of-function mutant for several generations ${ }^{16}$. Unlike the hrde-1 mutants that lost inheritance of oma-1 RNAi within two generations, effect of oma-1 RNAi knock-down persisted for 5-6 generations both in endu-2(lf) and wild type animals (Supplementary Fig. 6b), suggesting that ENDU-2 does not act in the nuclear RNAi pathway to control transgenerational inheritance of germline RNAi. Therefore, maintenance of germline immortality by ENDU-2 probably functions via a mechanism distinct from that of the nuclear RNAi pathway.

mRNA binding by ENDU-2, but not its mRNA-cleavage, is essential for maintaining germline immortality. ENDU-2 harbors two XendoU domains, of which the C-terminally localized domain is more similar to both human EndoU and Xenopus XendoU (Supplementary Fig. 7). It was suggested recently that ENDU-2 might also function as RNA-binding protein ${ }^{10}$. To identify the RNAs bound by ENDU-2, we precipitated ENDU-2:: EGFP and analyzed co-immunoprecipitated RNAs by deepsequencing (RIP-Seq). Since wild type ENDU-2 would potentially cleave its RNA targets, we thought that this might prevent enrichment of intact RNA targets and their subsequent identification. Although we had no direct proof of an RNA cleaving activity of ENDU-2 yet, we reasoned that generation of an ENDU2 variant that maintains RNA binding, but has lost RNA-cleavage activity, should facilitate RNA target detection. Xenopus XendoU mutants with $\mathrm{E}$ to $\mathrm{Q}$ exchanges at positions 161 or 167 of the EndoU domain lose RNA cleavage without reducing their RNAbinding activity ${ }^{17}$. The second glutamic acid (167E) is conserved in both XendoU domains of C. elegans ENDU-2 (175E and 460E), whereas the first $(161 \mathrm{E})$ is only found in the second XendoU domain (454E) (Supplementary Fig. 7b). We expressed both ENDU-2(E454Q)::EGFP and ENDU-2(E460Q)::EGFP variants in endu-2(lf) background and performed RIP-Seq with these two strains in additional to wild type ENDU-2::EGFP. By plotting normalized reads (RPM) of each transcript we were able to investigate RNA-binding affinity of ENDU-2 under different conditions. In general, all three ENDU-2 variants tested showed stronger RNA-binding affinity at $15^{\circ} \mathrm{C}$ than at $25^{\circ} \mathrm{C}$ (Fig. $4 \mathrm{a}$ ). E460Q showed weaker RNA-binding already at $15^{\circ} \mathrm{C}$ than wild type ENDU-2 and almost completely lost RNA-binding capacity at $25^{\circ} \mathrm{C}$. In addition, ENDU-2(E454Q) displayed stronger RNAbinding activity than ENDU-2(wt) at $15^{\circ} \mathrm{C}$. Therefore, we used RIP-Seq data of ENDU-2(E454Q) at $15^{\circ} \mathrm{C}$ for detecting RNAs 

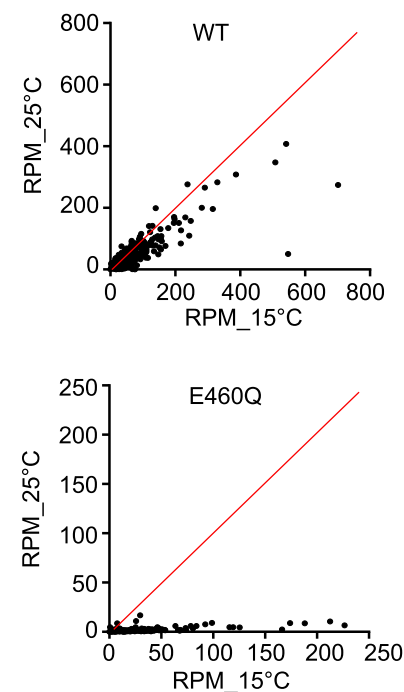

b

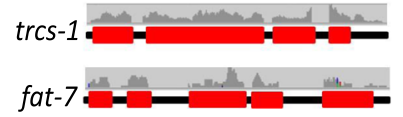

C

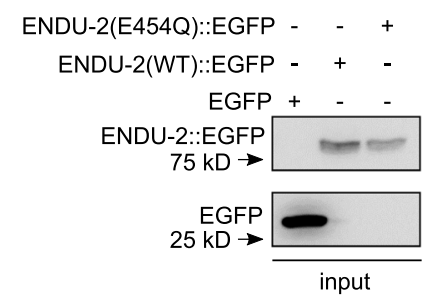

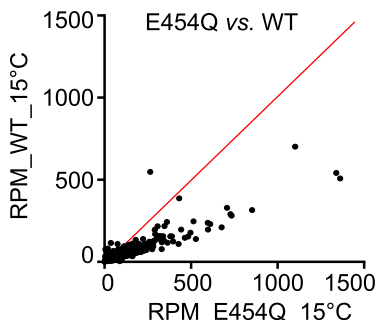

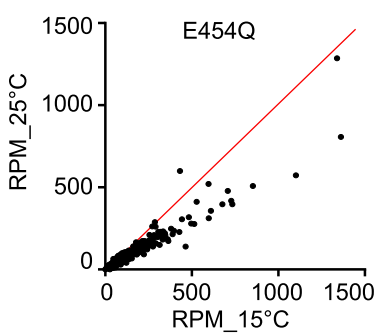

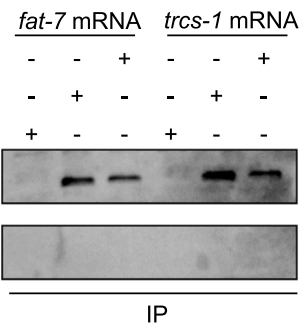

d

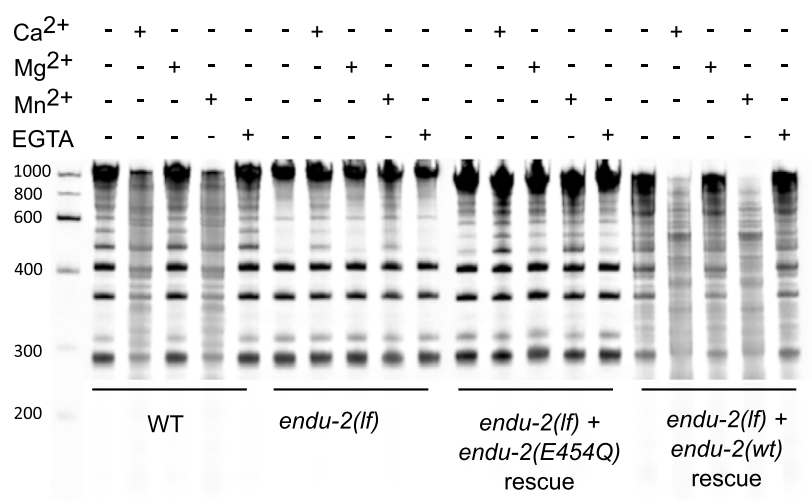

- endu-2(If) + endu-2 rescue transgene

\pm endu-2(If)

endu-2(E454Q)::EGFP endu-2(E460Q)::EGFP
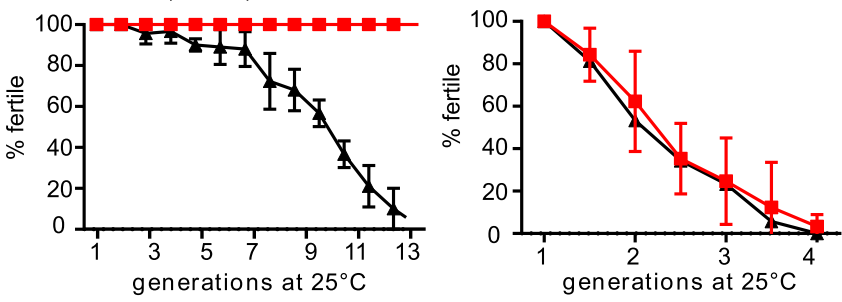

Fig. 4 RNA binding but not RNA-cleavage activity of ENDU-2 protects germline immortality. a Comparison of RNA binding activities of ENDU-2(wt): EGFP, ENDU-2(E454Q)::EGFP, and ENDU-2(E460Q)::EGFP at 15 and $25^{\circ} \mathrm{C}$. Shown are plotted normalized reads (RPM) from RIP-Seq of each identified transcript under different conditions. $\mathbf{b}$ Mapping of the RIP-Seq reads of two representative binding targets of ENDU-2. Only fragments of mRNA exons (red boxes) but not introns (black lines) were co-immunoprecipitated with ENDU-2. c ENDU-2::EGFP variants bind to selected mRNAs in vitro. Shown are western blots to detect proteins binding to fat-7 and trcs-1 mRNA, respectively. EGFP is a negative control. $N=3$ biological replicates. The uncropped blots are included in Supplementary Fig. 11b. d Wild type ENDU-2, but not ENDU-2(E454Q), leads to RNA decay (smear) in a Ca2+ and $\mathrm{Mn}^{2+}$ dependent manner, $N=3$. Shown is fused images of two gels from one experiment. The results of additional two biological replicates are shown in Supplementary Fig. 8a. e endu-2(E454Q)::EGFP but not endu-2(E460Q)::EGFP transgene rescues the Mrt phenotype. Data are mean $\pm S D, N=3$. Both endu-2(tm4977) and endu-2(tm4977) carrying endu-2(E454Q)::EGFP or endu-2(E460Q)::EGFP transgenes were decedents of one single PO animal carrying the respective transgenes.

bound by ENDU-2. 5920 transcripts were co-immunoprecipitated with ENDU-2(E454Q) (Supplementary Data 1). Most of them (>99\%) were protein-coding transcripts, the remaining were noncoding RNAs (5 snoRNAs, 27 pseudogenes, 5 ncRNAs, and 1 lincRNA). In addition, reads distribution analysis showed $>99 \%$ of sequenced reads were mapped to exons, suggesting that ENDU-2 (E454Q) primarily binds to processed mRNAs (Fig. 4b). We performed in vitro mRNA-binding assays with recombinant ENDU-2 proteins and two selected mRNA targets from the RIPSeq data and confirmed that both ENDU-2 and ENDU-2(E454Q) directly interact with these mRNAs (Fig. $4 \mathrm{c}$ and Supplementary Fig. 11b).

Studies of Xenopus XendoU had shown that that the RNA hydrolysis activity of XendoU requires $\mathrm{Mn}^{2+}$ or $\mathrm{Ca}^{2+18}$. ENDU2 seems to require similar conditions, since the addition of $5 \mathrm{mM}$ $\mathrm{Mn}^{2+}$ or $\mathrm{Ca}^{2+}$ in the buffer medium, but not $5 \mathrm{mM} \mathrm{Mg}^{2+}$, led to degradation of bulk RNA in the wild type worm lysates (Fig. $4 \mathrm{~d}$ and Supplementary Fig. 8). Bulk RNA degradation was strongly reduced in endu-2(lf) animals. In addition, expression of the wild type endu-2::EGFP transgene, but not of endu-2(E454Q)::EGFP, restored RNA decay in endu-2(lf) mutants, supporting our hypothesis that ENDU-2(E454Q) lost RNA-cleavage capacity despite its increased RNA-binding affinity. Surprisingly, extrachromosomal expression of ENDU-2(E454Q), but not of ENDU2(E460Q), fully rescued the Mrt phenotype of endu-2(lf) at $25^{\circ} \mathrm{C}$ (Fig. 4e). We conclude that RNA binding, rather than RNAcleavage activity of ENDU-2, is essential in the germline to maintain stem cell immortality at elevated temperature.

ENDU-2 negatively regulates somatic mRNA abundance via its endoribonuclease activity. To test if ENDU-2 affects mRNA levels, we performed microarray experiments at $25^{\circ} \mathrm{C}$ to determine alterations in the transcriptome mediated by ENDU-2 (procedure of sample preparation is illustrated in Supplementary Fig. 9a). A comparison of differential gene expression in 
endu-2(-) and endu-2(+) backgrounds suggested a similar number of transcripts being up- and downregulated by endu-2 (fold-change $>2$ ) (Fig. 5a). Among the 258 transcripts downregulated in endu-2(lf) animals, germline expressed genes $(n=$ 206) were over-represented (Supplementary Data 2). qPCR quantifications of two selected genes cav-1 and trsc-1 confirmed our microarray results and revealed that their expression levels strongly decreased in endu-2 mutants compared to wild type animals at $25^{\circ} \mathrm{C}$ (Supplementary Fig. 10c and 10d). However, neither a germline expressed cav-1::GFP reporter nor smFISH staining of trcs-1 confirmed significantly altered germline expression in situ (Supplementary Fig. 10a, 10b and 10d). As the gonads of endu-2(lf) mutants at $25^{\circ} \mathrm{C}$ became significantly smaller (Supplementary Fig. 9b), we speculate that apparent downregulation of germline mRNA within the transcriptome data set may be caused by reduced sample size of the gonad in endu-2 mutants.

Therefore, we put our focus on genes that were upregulated in endu-2(lf) background. Thirty-two percent (76 out of 237) of these transcripts were candidates for direct ENDU-2 targets, since

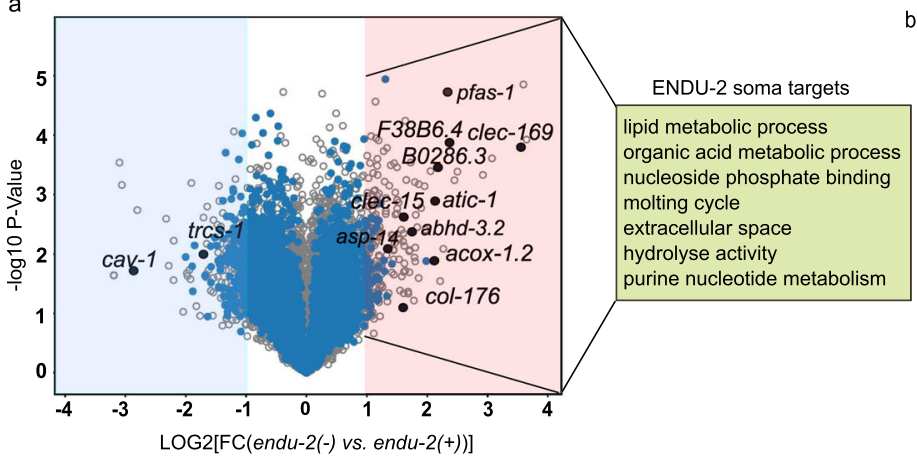

d

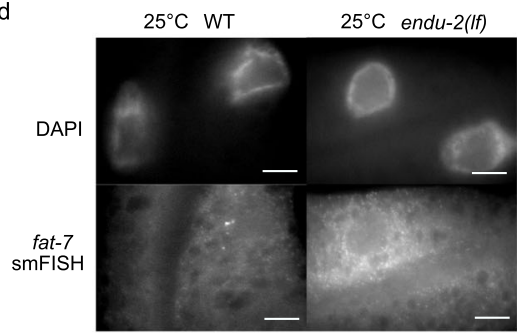

fat-7 mRNA

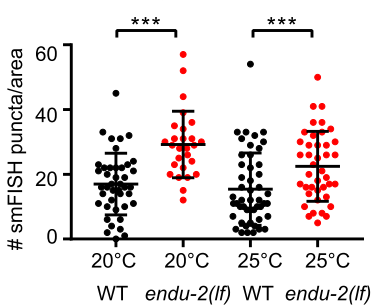

e
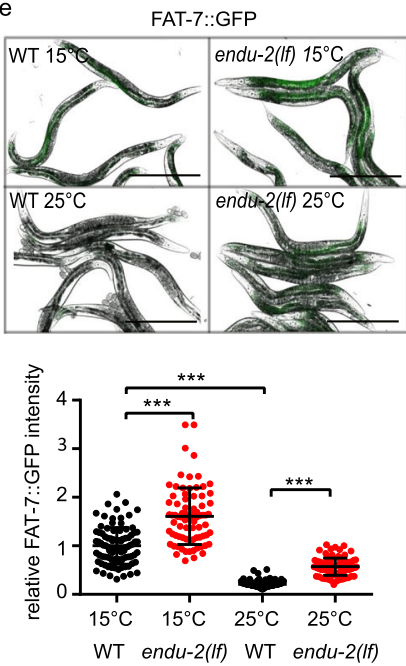

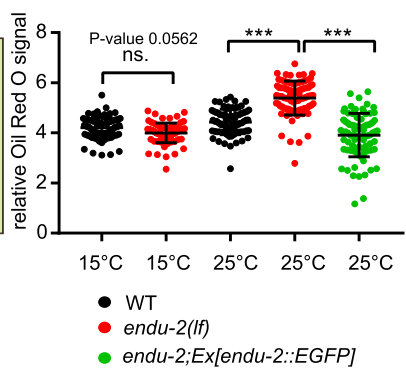

- endu-2(If)

- endu-2;Ex[endu-2::EGFP]

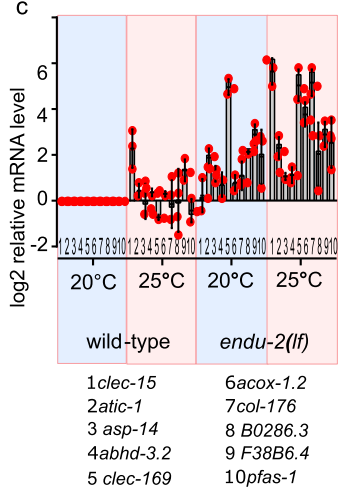

FAT-7::GFP $25^{\circ} \mathrm{C}$
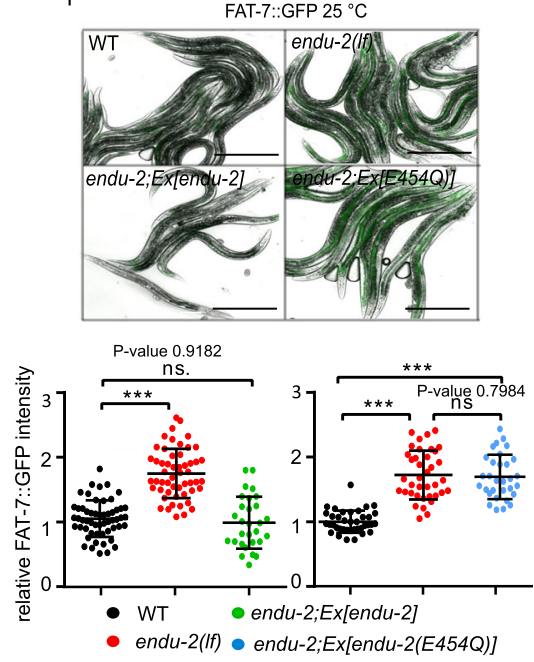

Fig. 5 ENDU-2 negatively regulates mRNA abundance in the soma. a Volcano blot of microarray results comparing endu-2(tm4977) mutant and endu-2 (tm4977) animals carrying the rescuing endu-2::EGFP transgene at $25^{\circ} \mathrm{C} . \mathrm{N}=3$ biological replicates. The dots in the bright red or blue areas are differentially regulated transcripts (FC $>2$ with two-tailed $P$-value $<0.05$ ). The blue dots mark transcripts bound by ENDU-2. Transcripts with blue and black label were ENDU-2 associated according to the RIP-Seq result and those with black labels were verified with qPCR. $\mathbf{b}$ ENDU-2 negatively regulates lipid content at $25^{\circ} \mathrm{C}$. Shown are mean \pm SD of quantification of relative Oil Red O stained signal in wild type, endu-2(tm4977) as well as endu-2(tm4977); byEx1375[endu-2::EGFP] day-one adult animals. Numbers of examined samples over three independent experiments: $n=80$ for wild type and endu-2 (tm4977) at $15^{\circ} \mathrm{C}$, wild type at $25^{\circ} \mathrm{C}, n=89$ for endu-2(tm4977) at $25^{\circ} \mathrm{C}, n=86$ for endu-2(tm4977); byEx 1375 [endu-2::EGFP] at $25^{\circ} \mathrm{C}$. Statistical test with one-way ANOVA. P-values were calculated with Tukey's multiple comparison test. $P$-value for WT vs. endu-2(tm4977) at $15^{\circ} \mathrm{C}: 0.0562$. ${ }^{\star \star \star} P$-value $<0.0001$. c qPCR to quantitate selected ENDU-2 target mRNAs in the soma at 20 and $25^{\circ} \mathrm{C}$. Data are mean $\pm \mathrm{SD}, N=$ three biological replicates. d Fluorescent images of smFISH staining of the ENDU-2 target fat-7 mRNA and quantification of the smFISH stained mRNA foci per examined area in wild type and endu-2(tm4977) animals. Scale bar $10 \mu \mathrm{m}$. Data are mean \pm SD. Numbers of examined samples over three independent experiments: wild type at $20^{\circ} \mathrm{C} n=42$, endu-2(tm4977) at $20^{\circ} \mathrm{C} n=31$, wild type at $25^{\circ} \mathrm{C} n=49$, endu-2(tm4977) at $25^{\circ} \mathrm{C} n=41$. Statistical test with one-way ANOVA. P-values were calculated with Tukey's multiple comparison test. ${ }^{\star \star \star} P$-value $<0.0001$. e Fluorescent images and quantification of fluorescence intensity of FAT-7: GFP in wild type and endu-2(tm4977) day-one adult animals at different temperatures. Data are mean \pm SD. Numbers of examined samples over three independent experiments: wild type at $15^{\circ} \mathrm{C} n=92$, endu-2(tm4977) at $15^{\circ} \mathrm{C} n=80$, wild type at $25^{\circ} \mathrm{C} n=102$, endu-2(tm4977) at $25^{\circ} \mathrm{C} n=89$. Statistical test with one-way ANOVA. $P$-values were calculated with Tukey's multiple comparison test. ${ }^{\star \star \star} P$-value $<0.0001$. Scale bar $500 \mu m$. f Fluorescent images and quantification of fluorescence intensity of FAT-7::GFP in wild type, endu-2(tm4977), endu-2(tm4977);Ex[endu-2(wt)] and endu-2 (tm4977); Ex[endu-2(E454Q)] day-one adult animals at $25^{\circ} \mathrm{C}$. Data are mean \pm SD. Numbers of examined samples over three independent experiments: For endu-2(wt) rescue experiment, wild type $n=55$, endu-2(tm4977) $n=53$, endu-2(tm4977);Ex[endu-2(wt)] $n=29$. For endu-2(E454Q) rescue experiment, wild type $n=38$, endu-2(tm4977) $n=38$, endu-2(tm4977);Ex[endu-2(E454Q)] $n=31$. Statistical test with one-way ANOVA. P-values were calculated with Tukey's multiple comparison test. P-value for WT vs. endu-2(tm4977);Ex[endu-2(wt)]: 0.9182, for endu-2(tm4977) vs. endu-2(tm4977);Ex[endu-2(E454Q)]: 0.7984. ${ }^{\star \star \star} P$-value $<0.0001$. Scale bar $500 \mu \mathrm{m}$. 
they had been co-immunoprecipitated with ENDU-2 (Supplementary Data 1). In addition, the vast majority of them (62 out of 76) did not have increased expression in the gonad of endu-2(lf) animals (see next chapter and Supplementary Data 1). This suggests that, at minimum, these 62 transcripts are downregulated by ENDU-2 in the somatic tissues. GO term analyses implicated these somatic ENDU-2 targets in regulation of various metabolic processes (Fig. 5a). Consistently, we found that loss of endu-2 resulted in increased lipid content at $25^{\circ} \mathrm{C}$ but not at $15^{\circ} \mathrm{C}$ (Fig. 5b). Taken together, these results indicate a putative regulatory role of ENDU-2 in limiting abundance of the mRNAs that are primarily involved in metabolic functions in the soma.

As some endu-2(lf) phenotypes were temperature-dependent, we asked whether ENDU-2 regulates mRNA abundance in response to temperature alterations. To test this, we performed qPCR to quantitate ten selected mRNAs that were bound and downregulated by ENDU-2. We found that mRNA levels of these targets were upregulated in endu-2 mutant background, compared to wild type, already at standard growth temperature $\left(20^{\circ} \mathrm{C}\right.$ ), and were even more abundant at $25^{\circ} \mathrm{C}$ (Fig. $5 \mathrm{c}$ ). Increased temperature, on the other hand, did not strongly affect the abundance of most of these mRNAs in wild type background. These results suggest a temperature-dependent negative influence of ENDU-2 on the levels of these mRNA targets. We also performed smFISH to inspect the influence of ENDU-2 on the mRNA level of yet another target fat-7. fat-7 mRNA was only detected in the intestine and endu-2(-) animals had higher fat-7 transcript levels than wild type at both 20 and $25^{\circ} \mathrm{C}$ (Fig. $5 \mathrm{~d}$ ). Moreover, we used a fat-7::GFP translational fusion reporter to monitor FAT-7::GFP protein level. Wild type animals showed reduced FAT-7::GFP levels at $25^{\circ} \mathrm{C}$ vs. $15^{\circ} \mathrm{C}$ (Fig. 5e). endu-2(lf) displayed stronger FAT-7::GFP expression levels at both temperatures. Furthermore, transgenic expression of endu-2(wt) but not the endu-2(E454Q) transgene (that has lost RNA-cleavage activity) restored decreased FAT-7::GFP level (Fig. 5f). We conclude that ENDU-2 mediated mRNA-cleavage is required for decreasing expression of at least some of its somatic target genes, such as fat-7.

ENDU-2 prevents misexpression of soma-specific genes in the germline. To investigate germline transcriptomes regulated by ENDU-2, we isolated gonads from wild type and endu-2(lf) animals which had been grown at $25^{\circ} \mathrm{C}$ from L1 stage, and sequenced two biological replicates of polyadenylated RNA from each strain. A gene with normalized reads of RPKM $>1$ was scored as being expressed. We identified 8356 expressed genes in the gonad of wild type animals at $25^{\circ} \mathrm{C}$, among which $88 \%$ (7365) have been reported as expressed in the wild type gonad at $20^{\circ} \mathrm{C}^{19}$. The following DESeq2 analysis showed that upregulation of mRNA levels was the prevalent change in the gonad of endu-2 mutants (422 upregulated, 35 downregulated, FC $>2, P$-value $<0.05$; Fig. 6a, Supplementary Data 3 ). Only genes upregulated in endu-2(lf) background were enriched in ENDU-2 binding, as 218 out of 422 upregulated genes ( $P$-value $<0.0001$, Chi-square test) and 11 out of 35 downregulated genes $(P$-value $=0.7675$, Chisquare test) were co-immunoprecipitated with ENDU-2. We considered them as direct targets of ENDU-2 and grouped them in two classes, depending on whether they are up- (class I) or downregulated (class II) in endu-2(lf) background (Fig. 6b). Expression of a class I targets is repressed, while class II genes are activated by wild type ENDU-2 activity. Consistently, we noticed that most of the class I, but not the class II target genes, were not, or very lowly, expressed in the gonad of wild type animals (Fig. 6c). Additional tissue enrichment analysis suggested that the class I targets are enriched in genes expressed in the somatic tissues (intestine, neurons, muscles, somatic gonad, and pharynx). GO term enrichment analysis revealed that the major function of class I targets are involved in immune and defense responses (Fig. 6b). We wondered whether the class I target genes were preferentially soma-specific genes that are misexpressed in the germline in the absence of ENDU-2. To validate this assumption, we combined our wild type gonad transcriptome data with a previous published whole animal's transcriptome analysis at $25^{\circ} \mathrm{C}^{20}$ and calculated a soma enrichment factor (SEF) for each transcript to estimate the expression ratio between the soma and the gonad. Class I, but not class II, targets had on average significantly higher SEF values than the total mRNAs (Fig. 6d), suggesting that the class I genes are predominantly expressed in the soma, but only expressed at low levels in the germline of wild type animals. As maintenance of germline immortality by ENDU2 does not require its RNA-cleavage activity, we additionally sequenced mRNA from isolated gonads of endu-2(lf) mutant carrying the endu-2(E454Q)::EGFP transgene. Expressing endu-2 (E454Q)::EGFP was sufficient to repress germline expression of most class I targets (Fig. 6e and f). In summary, our data indicate that the inhibitory role of ENDU-2 to prevent germline expression of soma-specific genes is probably independent of its RNA-cleavage activity.

\section{Discussion}

The task of the germline is to maintain pluripotency and immortality for an accurate transmission of genetic and epigenetic information between generations. Despite emerging studies about molecular mechanisms protecting the germline from the impacts of stress, one obvious open question is whether the germline responds directly to environmental signals, such as nutrients or stress, or rather reacts to alterations in somatic signaling. A direct stress response seems to be less advantageous from an economical point of view, since this requires, in a pluripotent stem cell, the expression of an entire hierarchy of mechanisms, involving the sensation of stress, signaling, as well as responsive pathways to enable a dynamic and rapid response to a wide range of environmental cues. In contrast, the second alternative would be more beneficial, as differentiated somatic cells are specialized to sense environmental changes. In such a case, molecular messengers, such as secreted hormones and ligands, are required to shuttle between somatic and germline tissues to adjust a proper response in the germline. Previous studies have revealed that microRNAs could act as such messengers mediating communication between soma and germline ${ }^{21,22}$. Here, we show that secretion of a conserved endoribonuclease ENDU-2 from the soma prevents misexpression of soma-specific gene in the germline and preserve germline immortality at elevated temperature. This finding, together with another recent study reporting regulation of germline proliferation by intestinal ENDU-2 in response to thymidine imbalance ${ }^{11}$, strongly suggests ENDU-2 as a crucial molecule mediating non-cell-autonomous stress responses.

Our data implicate ENDU-2 in enabling long-range communication between cells, mediated via its secretion signal peptide (Fig. 2b). Whereas an earlier study had claimed neurons and muscles as the tissues primarily expressing endu-2 $2^{10}$, our data, consistent with the report from Jia et al., suggest intestine as the most important organ to produce ENDU-2, whereas expression in neuronal, muscular, and somatic gonad tissues is rather weak (Fig. 2a and Supplementary Fig. 3). We compared, for this analysis, both RNA and protein levels of ENDU-2 as well as different ENDU-2 variants with truncations of the $\mathrm{N}$-terminal secretion signal peptide $\left(\Delta_{\mathrm{ss}}\right.$ ENDU-2 and $\mathrm{SS}_{\text {sel- } 1}:: \Delta_{\mathrm{ss}}$ ENDU-2). We could demonstrate that the tissues containing ENDU-2 protein differ 


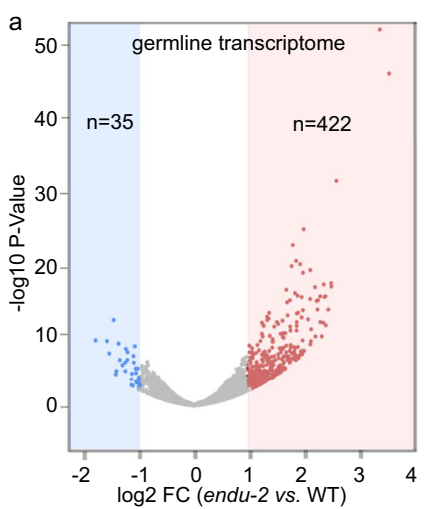

b

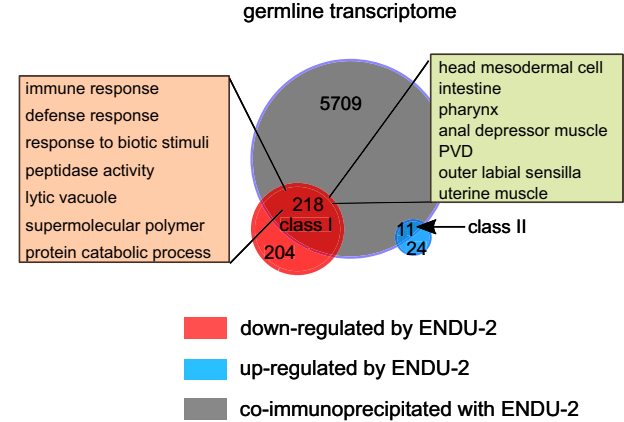

germline mRNA

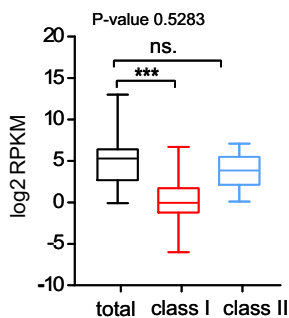

d

relative expression soma/germline P-value 0.1477

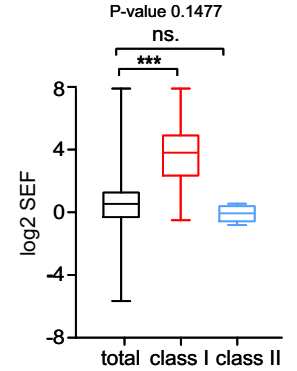

e

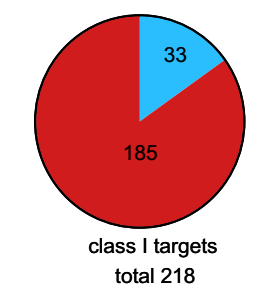

not rescued with endu-2(E454Q) rescued with endu-2(E454Q)

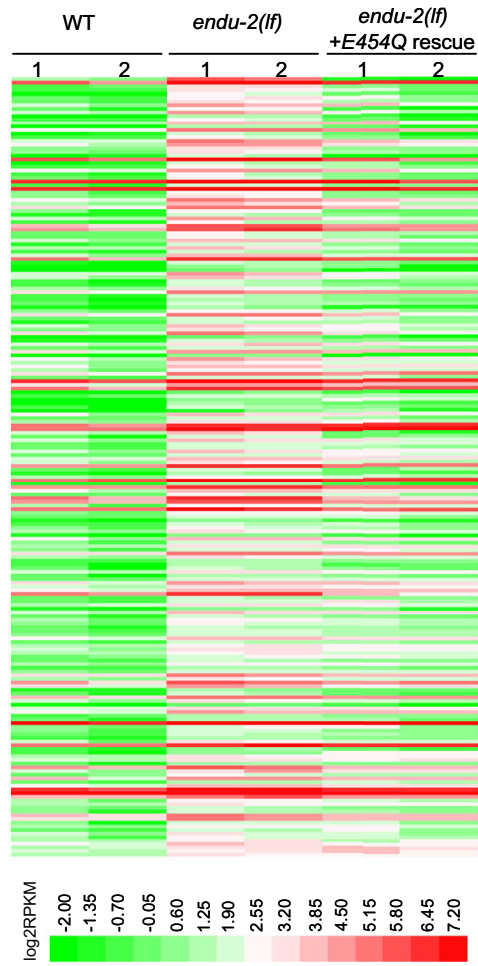

Fig. 6 ENDU-2 prevents misexpression of soma-specific genes in the germline. a Volcano plot of gonadal expressed genes in endu-2(tm4977) relative to wild type day-one adult animals grown up at $25^{\circ} \mathrm{C}$. Two biological replicates were obtained for each strain. Significantly misregulated genes (FC $>2$, FDR $<$ 0.05) were calculated with DESeq2 and are shown in red or blue. $n$ refers to the total numbers of up- or downregulated genes. $\mathbf{b}$ Comparison of the total transcripts co-immunoprecipitated with ENDU-2 and ENDU-2 regulated genes in the gonad. c Comparison of expression levels (RPKM) of total gonadexpressed genes $(n=8356)$, ENDU-2 class I $(n=218)$ and class II targets $(n=11)$ in wild type animals. Shown are boxes extending from the 25 th to the 75th percentile, with the median indicated by the horizontal line and Min/Max whiskers. Statistical test with one-way ANOVA. $P$-values were calculated with Dunnett's multiple comparison test. $P$-value for total vs. class II targets: 0.5283 . ${ }^{\star \star \star} P$-value $<0.0001$. d Comparison of relative expression between the soma and the gonad (SEF) of the total transcripts $(n=12371)$, ENDU-2 class I $(n=218)$, and class II ( $n=11)$ targets in wild type animals. Shown are boxes extending from the 25th to the 75th percentile, with the median indicated by the horizontal line and Min/Max whiskers. Statistical test with one-way ANOVA. $P$-values were calculated with Dunnett's multiple comparison test. $P$-value for total vs. class II targets: 0.1477 . ${ }^{\star \star \star} P$-value $<0.0001$. e The majority of the alerted expression of the germline class I targets is rescued by endu-2(E454Q)::EGFP transgene. $\mathbf{f}$ Heat map of ENDU-2 repressed germline genes in wild type, endu-2(tm4977) and endu-2;Ex[endu-2(E454Q)::EGFP] animals.

from those that harbor endu-2 mRNA and presence of a secretion signal peptide is necessary and sufficient to target ENDU-2 to the secretory pathway to reach distant cells.

Our data suggest that ENDU-2 production and function may occur in different tissues, and that secretion of ENDU-2 allows the control of mRNA abundance in the distance. A simple assumption would be that ENDU-2 has similar functions in any of its target tissues, no matter from which cells it is expressed. However, only neuronally or intestinally expressed ENDU-2 was able to rescue germline immortality (Fig. 3c), indicating existence of functional difference of ENDU-2 from distinct origins. Tissuespecific interactors or modifiers of ENDU-2 are probably crucial to specify its individual activities. It is currently not known whether there are isoform-specific functions of ENDU-2, or whether ENDU-2 protein activity is modulated by protein modifications or interactions.

We distinguished two activities of ENDU-2: mRNA binding and mRNA-cleavage. RIP-Seq and transcriptomic results demonstrated that the levels of only about $10 \%$ of the mRNAs to which ENDU-2 binds changed in endu-2(lf) mutants at elevated temperature (Figs. 4-6), suggesting that ENDU-2 is able to discriminate whether its targets are only bound or are hydrolyzed. Such a scenario has also been proposed for SMG-2/UPF1, the core factor of the non-sense mediated decay (NMD) complex that degrades only a small fraction of the RNAs it binds to ${ }^{23}$. In addition, binding to mRNA might generate a platform for interaction with other RNA-binding proteins which may act together with ENDU-2 to regulate RNA stability. ENDU-2 represses expression of its somatic target genes probably via direct RNA degradation, while it utilizes RNA binding, but not cleavage activity, to prevent misexpression of soma-specific genes in the germline, suggesting rather an indirect mechanism of ENDU-2 in the germline to prevent a gene expression program specific for differentiated somatic cells. Whether this is regulated at transcriptional or post-transcriptional level is currently unknown. Another puzzle is how germline localized ENDU-2 selectively represses expression of soma-specific genes. Germ granules of $C$. elegans have been shown to inhibit misexpression of somatic transcripts ${ }^{24}$. The punctate localization pattern of ENDU-2 in the germline indicates its possible localization in the germ granules (Fig. 2c). Whether ENDU-2 affects functions of the germ granules to restrict somatic transcripts should be studied in the future. We speculate that association of ENDU-2 with distinct protein complexes in the soma and germline may differentiate its functionalities and adjust gene expression in the soma and germline in response to temperature alterations.

Xenopus EndoU was suggested to both control snoRNA biogenesis in the nucleolus and cleave mRNAs in the cytosol ${ }^{17,18}$. 
Mouse EndoU has been reported to regulate peripheral B cell survival via reducing c-Myc mRNA level ${ }^{25}$. Inactivation of neuronal Drosophila DendoU causes neurotoxicity partially by downregulation of dTDP-4326. SARS and SARS-Covid-2 EndoU homologs Nps15/NendoU have been implicated in virus replication as well as limitation of host innate immune response, although the detailed functions are still enigmatic ${ }^{27}$. Our work here suggests that $C$. elegans ENDU-2, in response to environmental stimuli such as temperature elevation, can exert its function in tissues that different from those expressing it. Therefore, ENDU-2 is a previously undescribed candidate for transmitting environmental signals across tissue boundaries, most notably involving signaling to the germline. A secretion signal peptide is also present in the human EndoU homolog that is expressed in the placenta and detected in the serum ${ }^{28}$. Therefore, the potential conservation of its non-cell-autonomous functions could open a research area to study intercellular communication. EndoU, thus, could be involved in a mechanism by which environmental influences and experiences of the somatic tissues are transmitted into the reproductive system.

\section{Methods}

Strains. The C. elegans N2 (Bristol) strain was used as wild type in all experiments in this study. Mutant strains: KHR83 endu-2(tm4977), outcrossed 8x with our N2. BR7130 endu-2(by188), NL3511 ppw-1(pk1425), TX20 oma-1(zu405), BR7510 oma1(zu405);endu-2(tm4977), BR8649 oma-1(zu405);hrde-1(tm1200), BR7205 endu-2 (by190[endu-2::EGFP]), BR7089 byEx1315[endu-2P::EGFP;rol-6(su1006)], BR8657 endu-2(tm4977);byEx1814[endu-2P::endu-2::EGFP::endu-2 $\left.3^{\prime} U T R ; r o l-6(s u 1006) ;\right]$, BR7295 endu-2(tm4977); byEx1375[endu-2P::endu-2::EGFP;myo-2P::mCherry], BR5402 byEx749[unc-119P::EGFP;rol-6(su1006)], BR8672 byEx1821[unc-119P::

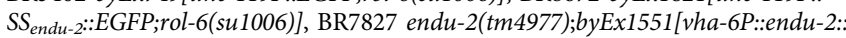
EGFP::3xFlag;myo-2P::mCherry], BR7332 endu-2(tm4977);byEx1379[fos-1P::endu2::EGFP;myo-2P::mCherry], BR8551 endu-2(tm4977);byEx1795[unc-119P::endu-2:: EGFP::3xFlag;rol-6(su1006)], BR8662 endu-2(tm4977);byEx1816[myo-3P::endu-2:: EGFP::3xFlag;myo-2P::mCherry], BR7512 endu-2(tm4977); byEx1449[endu-2P:: $\Delta_{s s}$ endu-2::EGFP;rol-6(su1006);myo-2P::mCherry], BR8747 endu-2(tm4977); byEx1847[endu-2P:: $\Delta_{\text {ss }}$ endu-2::EGFP:: endu-2 $\left.3^{\prime} U T R ; m y o-2 P:: m C h e r r y\right]$, BR8821 endu-

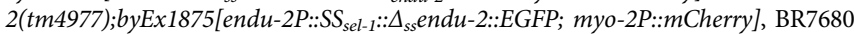
endu-2(tm4977); byEx1492[endu-2P::endu-2(E454Q)::EGFP::3xFlag;myo-2P:: $m$ Cherry], BR7683 endu-2(tm4977);byEx1495[endu-2P::endu-2(E460Q)::EGFP::3xFLAG;myo-2P::mCherry], DMS303 nIs590[fat-7p::fat-7::GFP + lin15(+)], BR8317 endu-2(tm4977);nIs590[fat-7p::fat-7::GFP + lin-15(+)], BR8754 endu-2(tm4977); nIs590[fat-7p::fat-7::GFP + lin-15(+)];byEx1853[endu-2P::endu-2(E454Q);myo-2P:: $m$ Cherry], BR8775 endu-2(tm4977);nIs590[fat-7p::fat-7::GFP + lin-15(+)];byEx1860 [endu-2P::endu-2;myo-2P::mCherry], RT688 unc-119(ed3);pwIs28[pie-1p::cav-1::GFP (7)+unc-119(+)], BR8358 endu-2(tm4977);pwIs28[pie-1p::cav-1::GFP(7)+unc-119 $(+)$ ]. For RIP-Seq the following strains were used: BR7802 endu-2(tm4977);byIs240 [endu-2P::endu-2(E460Q)::EGFP::3xFLAG;myo-2p::mCherry], BR7803 endu-2 (tm4977);byIs241[endu-2P::endu-2(E454Q)::EGFP::3xFLAG;myo-2P::mCherry], BR7205 endu-2(by190[endu-2::EGFP]) and Ex[ife-2P::GFP] (as IP control, gift from Tavernarakis Lab). For the RNA-cleavage assay, BR8311 endu-2(tm4977);byIs267 [endu-2P::endu-2::EGFP;myo-2::mCherry] and BR7803 endu-2(tm4977);byIs241 [endu-2P::endu-2(E454Q)::EGFP::3xFlag;myo-2P::mCherry] were used in additional to N2 and endu-2(tm4977) strains. endu-2(tm4977) allele was used for all experiments, if not noted otherwise. Except for the Fig. 1a and Supplementary Fig. 2, all the endu-2(lf) mutants were the granddaughter generation (G2) from an endu-2 (tm4977) carrying transgenic endu-2 rescue strains whose parents (G1) had lost the rescue transgene. Information for extrachromosomal transgenic strains generated in this study is summarized in the Supplementary Data 5.

Plasmids. To construct an EGFP transcriptional fusion reporter of endu-2 (pBY3798), a 4691 bp genomic fragment upstream of the endu-2 ATG was PCR amplified and inserted into pEGFP-N1 with Eco47III/BglII sites. For translational fusion reporters, 5487 bp endu-2 genomic region was cloned into pBY3798 with BglII/SmaI sites to receive endu-2P::endu-2::EGFP (pBY3800), the endu-2 $3^{\prime} \mathrm{UTR}$ was inserted into pBY3800 at NotI site to receive endu-2P::endu-2::EGFP:: endu-2 3 UTR (pBY4137). $\Delta_{s s}$ endu-2::EGFP (pBY3843) and $\Delta_{s s}$ endu-2::EGFP:: endu-2 $3^{\prime} U T R$ construct (pBY4172) was generated by removing sequences encoding the $\mathrm{N}$ terminal 2-19 amino acids from pBY3800 and pBY4137, respectively. The endu$2 P:: S S_{\text {sel- } 1}: \Delta_{s s}$ endu-2::EGFP construct (pBY4194) was generated by inserting the sequence encoding the amino acids 1-20 of sel-1 into the BglII site of pBY3843. A endu-2P::3xFlag::endu-2::EGFP:: endu- $2^{\prime} 3^{\prime} U T R$ expressing construct (pBY4138) was generated by inserting $3 \times$ Flag encoding sequence and endu- 2 genomic region into pBY3798 with BglII and BglII/SmaI sites, respectively. The $3^{\prime} \mathrm{UTR}$ of endu-2 was inserted via Gibson ligation. The unc-119P::EGFP expressing construct (pBY2941) was made by fusing the $2.1 \mathrm{~kb}$ unc-119 promoter region into pEGFP-N1 with

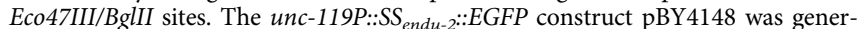
ated by inserting the first 57 nucleotides of endu- 2 cDNA into pBY2941 with BglII/ AgeI sites. For expressing recombinant ENDU-2::EGFP in HEK 293T cells, wild type endu-2 cDNA was cloned into a 3xFlag containing pEGFP-N1 with Xhol/ SmaI (pBY3878). Site-directed mutagenesis was performed to receive endu-2 (E454Q)::EGFP::3xFlag (pBY3894) and endu-2(E460Q)::EGFP::3xFlag mutants (pBY3895). The fragments endu-2::EGFP::3xFlag from $p B Y 3878$, endu-2(E454Q):: EGFP::3xFlag from pBY3894 and endu-2(E460Q)::EGFP::3xFlag from pBY3895 were inserted into pBY3798 to receive constructs for generating endu-2P::endu-2: EGFP::3xFlag (pBY3892), endu-2P::endu-2(E454Q)::EGFP::3xFlag (pBY3897) and endu-2P::endu-2(E460Q)::EGFP::3xFlag (pBY3898) reporter strains. EGFP::3xFlag fragments in pBY3892 and pBY3897 were removed to receive pBY4188 endu-2P: endu-2 and pBY4066 endu-2P::endu-2(E454Q). Somatic gonad specific expression was achieved via PCR amplifying and insertion of endu-2 cDNA into a fos-1P::GFP plasmid (a gift from David Sherwood lab) with SalI and SmaI sites (pBY3833). For intestinal specific expression, a $1.2 \mathrm{~kb}$ vha-6 promoter was inserted at Eco47III/ XhoI sites into pBY3878 to receive vha-6P::endu-2::EGFP::3xFlag (pBY3937). A 2.1 $\mathrm{kb} u n c-119$ promoter was inserted into $\mathrm{pBY} 3878$ at Eco47III/BglII sites to receive unc-119P::endu-2(cDNA)::EGFP::3xFlag (pBY4127) for neuronal expression. A 2.4 $\mathrm{kb}$ myo-3 promoter region was cloned into $\mathrm{pBY} 3878$ to receive myo-3P::endu-2:: EGFP::3xFlag (pBY4135) for muscular specific endu-2 expression.

Scoring of Mrt phenotype. Mrt phenotype was examined by quantification of brood size by separating 15 L4 animals on single plates and counting number of progeny per animal, or transferring six L4 larvae onto new agar plates and scoring percentage of the fertile animals $36 \mathrm{~h}$ after the $\mathrm{L} 4$ stage in the next generation. Both endu-2(tm4977) and endu-2(by188) were further outcrossed $4 \times$ with N2 before scoring Mrt phenotype for Fig. 1a and Supplementary Fig. 2a. For brood size recovery at $15^{\circ} \mathrm{C}$, wild type and outcrossed endu-2(tm1977) animals were maintained at $25^{\circ} \mathrm{C}$ for 6 generations until endu-2(tm4977) showed strong sterile phenotype. Fifteen L1-L2 animals from the 6th generation were shifted to $15^{\circ} \mathrm{C}$ and designated as G1. For Mrt rescue experiment, endu-2(tm4977) progenies of endu-2(tm4977);byEx1375[endu-2::EGFP;myo-2P::mCherry], endu-2(tm4977); byEx1449[endu-2P:: $\Delta_{s s}$ endu-2::EGFP;myo-2P::mCherry], endu-2(tm4977);byEx1875

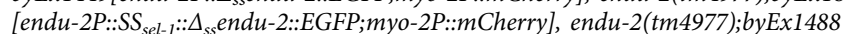
[endu-2P::endu-2(E454Q)::EGFP::3xFLAG;myo-2P::mCherry] and endu-2(tm4977); byEx1488[endu-2P::endu-2(E460Q)::EGFP::3xFLAG;myo-2P::mCherry] animals (P0) were isolated and designated as G1.

Germline proliferation. The 'number of nuclei in the proliferative zone' included all the germ nuclei between the distal tip and the transition zone of day-one adult animals ( $24 \mathrm{~h}$ after mid-L4 larval stage). For visualizing and counting germ cell nuclei in the proliferating zone of the gonad, dissected gonads were fixed in methanol and suspended in PBST containing $0.1 \%$ Tween 20 and $2 \mu \mathrm{g} / \mathrm{ml} \mathrm{4} 4^{\prime}, 6-$ diamidino-2-phenylindole (DAPI) before microscopy. Z-stack images of animals were collected and the numbers of the germ cells were counted with a publicly available ImageJ Cell Counter plug-in originally written by Kurt De Vos at the University of Sheffield, Academic Neurology.

Lifespan. Lifespan at $20^{\circ} \mathrm{C}$ was initiated at L4 stage. As endu-2(tm4977) animals display strong egg-laying defect due to abnormal vulval development, agar plates containing $200 \mu \mathrm{M}$ FUDR were used to during the first seven days of adulthood to avoid internal hatching.

Antibody staining. Antibody staining of dissected gonads was performed with day-one adult animals ${ }^{29}$. A 1:200 dilution of a monoclonal mouse anti-GFP antibody (Roche, Nr. 11814460001) and a 1:25 dilution of the secondary antibody were used to detect ENDU-2::EGFP in the gonad.

oma-1 RNAi inheritance assay. oma-1 RNAi was initiated at L1 stage at $20^{\circ} \mathrm{C}$ (restrictive temperature) and these animals were designated as P0. Six P0 day-one adults animals were transferred onto OP50 seeded agar plates and incubated at $20^{\circ} \mathrm{C}$ for scoring the embryonic lethality over generations. Embryonic lethality was examined by transferring of about 100 embryos onto an OP50 seeded agar plate and quantifying the number of hatched animals after $48 \mathrm{~h}$.

Oil Red $\mathrm{O}$ staining to quantify content of body fat. Worms were fixed in $500 \mu$ $60 \%$ isopropanol. After removal of supernatant, $500 \mu$ freshly filtered ORO working solution was added to stain worms at $25^{\circ} \mathrm{C}$ in a wet chamber overnight. Animals were washed once with $1 \mathrm{ml} 0.01 \%$ Triton-x100 containing M9 buffer and responded in $250 \mu \mathrm{l} \mathrm{M9-Triton-x100} \mathrm{buffer.} \mathrm{Stained} \mathrm{worms} \mathrm{can} \mathrm{be} \mathrm{stored} \mathrm{at} 4{ }^{\circ} \mathrm{C}$ for at least 1 month. For quantification, color images were recorded. The original images were spitted into red and blue channels and the level of Oil Red-O was quantified by determining the excess intensity in the red channel in comparison to the blue channel. The mean lipid content per worm was calculated as the total intensity within stained regions normalized by the area of the worm regions. Oil Red O working solution was prepared as follows: $0.5 \mathrm{~g}$ of Oil Red O powder was 
dissolved in $100 \mathrm{ml}$ isopropanol solution and equilibrated for several days. The solution was then freshly diluted with $40 \%$ water for a $60 \%$ stock and allowed to sit overnight at room temperature and filtered using $0.2 \mathrm{~mm}$ filters.

RIP-Seq. To isolate ENDU-2 associated RNAs, animals of mixed stage were lysed in Lysis-NP-40 buffer ( $150 \mathrm{mM} \mathrm{NaCl}, 50 \mathrm{mM}$ Tris pH 8.0, 1\% NP-40 + Protease Inhibitor $+100 \mu \mathrm{l} / \mathrm{ml}$ RNase Inhibitor) with SilentCrusher $S(2 \times 30 \mathrm{~s} 12,600 \times g)$. Lysates were clarified by centrifuging at $16,000 \times g$ for $15 \mathrm{~min}$. Supernatants were pre-cleared with Dynal Protein A magnetic beads (Invitrogen) and incubated with anti-GFP antibody (Abcam) for $1 \mathrm{~h}$ at $4^{\circ} \mathrm{C}$, followed by incubation with Dynal Protein A Magnetic Beads for $2 \mathrm{~h}$ at $4{ }^{\circ} \mathrm{C}$. Beads were washed three times with Lysis-NP-40 Puffer before DNase I digestion ( $100 \mu \mathrm{l}$ 1x DNase buffer, $1 \mu \mathrm{l}$ DNase I, $1 \mu \mathrm{l}$ RNase inhibitor) at $37^{\circ} \mathrm{C}$ for $30 \mathrm{~min}$. In all, $10 \mu \mathrm{l}$ of $50 \mathrm{mM}$ EDTA was added to stop DNase digestion. RNA was extracted with 5 volumes Trizol reagent (Invitrogen), followed by isopropanol precipitation. Precipitates were resolved in RNase-free water for visualization on $12.5 \%$ denaturing acrylamide gel with SYBR Gold (Thermo Scientific). Random hexamer primers were used for preparing TruSeq 3 reverse-stranded cDNA libraries for Illumina single-end 50 bp sequencing. RIP-Seq to compare RNA-binding efficiency of ENDU-2(wt), ENDU-2 (E454Q), and ENDU-2(E460Q) at 15 and $25^{\circ} \mathrm{C}$ were performed without crosslinking. RIP-Seq to determine RNA targets of ENDU-2 was carried out with ENDU-2(E454Q)::EGFP expressing animals raised at $15^{\circ} \mathrm{C}$ with UV cross-linking.

Analysis of RIP-Seq data. The sequencing data were uploaded to the European Galaxy Server at https://usegalaxy.eu, and all read quality controls, trimming, mapping and counting were performed through Galaxy ${ }^{30}$. Specifically, after trimming with Trimmomatic $0.36^{31}$, we mapped the reads to the C. elegans genome using the Wormbase WS260 genomic sequence (ftp://ftp.wormbase.org/pub/ wormbase/releases/WS260/species/c_elegans/PRJNA13758/c_elegans. PRJNA13758.WS260.genomic.fa.gz) and the WS260 canonical gene set (ftp://ftp. wormbase.org/pub/wormbase/releases/WS260/species/c_elegans/PRJNA13758/ c_elegans.PRJNA13758.WS260.canonical_geneset.gtf.gz) with the RNA aligner STAR 2.6.0 $\mathrm{b}^{32}$ with default settings, then counted reads mapping to individual genes using feature Counts $1.6 \cdot 2^{33}$. For a read to be counted as mapping to a particular gene, we required a minimum read mapping quality of 12 (-Q 12 option of feature Counts) and an overlap of at least 1 base between the read and any of the exons of the gene (--minOverlap 1).

The IP enrichment of a transcript was expressed as $\mathrm{FC}=(\mathrm{IP} \mathrm{RPM}+1) /($ Mock IP RPM +1 ), where RPM = number of reads mapped to a gene/(number of all mapped reads) $\times 10^{6}$. The addition of 1 in the FC formula prevents infinite enrichment. ENDU-2 associated RNAs were defined as transcripts with both FC > $=4$ and $\mathrm{RPKM}>=1$ in ENDU-2(E454Q) IP sample. RPKM is calculated as follows: $\mathrm{RPKM}=\mathrm{RPM} /$ length of transcripts $\times 10^{3}$.

RNA-seq and data processing of isolated gonads. Animals were raised at $25^{\circ} \mathrm{C}$ from the L1 larval stage and the complete gonads were isolated $24 \mathrm{~h}$ after mid-L4 stage. Total RNA of the gonads was extracted with RNeasy ${ }^{\circledR}$ Mini Kit (Qiagen, Venlo, The Netherlands). Purification of poly-A containing RNA molecules, RNA fragmentation, strand-specific random primed cDNA library preparation, and single-read sequencing (50 bp) on an Illumina HiSeq 4000 were carried out by Eurofins Genomics. The RNA-seq results from two independent biological replicate of dissected gonads were uploaded to the European Galaxy Server at https:// usegalaxy.eu. All read quality controls, trimming, mapping and counting were performed through Galaxy ${ }^{30}$ using protocols like described for RIP-Seq (above). The DESeq2 (Galaxy Version 2.11.40.6 + galaxy1) was used to determine differentially expressed features from count tables of differential transcript abundances. The RPKMs throughout this study were normalized separately. Only the longest isoform of each gene was used as length of a transcript. To estimate genes with enriched expression in the soma, RNA-seq data from wild type animals raised at $25^{\circ} \mathrm{C}$ from a previous study ${ }^{20}$ were compared with our transcriptome from isolated gonads of wild type animals. The soma enrichment factor (SEF) was calculated with the formal SEF $\left.=\left(\mathrm{RPKM}_{\text {whole animal }}+2\right) /\left(\mathrm{RPKM}_{\text {onad }}+2\right)\right]$. Addition of factor 2 to the formal minimize large SEF value caused by low RPKM values.

In vitro RNA-binding assay of recombinant ENDU-2 protein. The RNA-binding assay was performed as described ${ }^{34}$. fat 7 and trcs-1 mRNA were transcribed from complementary DNA (cDNA) with biotinylated uracil. The presence and purity of RNA were examined on an RNA gel. This biotin-labeled RNA was incubated with recombinant ENDU-2(wt):::EGFP::3xFlag, ENDU-2(E454Q)::EGFP::3xFlag, and ENDU-2(E460Q)::EGFP::3xFlag proteins expressed in HEK 293T cells. The labeled RNA was captured and isolated using streptavidin beads. Proteins binding the RNA were then visualized via western blot. The uncropped blots are included in the Supplementary Fig. 11.

RNA-cleavage assay. Pellets of animals raised at $15^{\circ} \mathrm{C}$ in mixed stages were washed twice with $1 \mathrm{ml} 10 \mathrm{mM}$ EGTA containing M9 buffer before resuspension in $0.7 \mathrm{ml} \mathrm{NP}-40$ buffer. $3 \mathrm{x}$ Protease inhibitor was added to the buffer and worms were lysed with SilentCrusher $(2 \times 30,12,600 \times g) .5 \mu \mathrm{l}$ RNAsin Plus RNAse Inhibitor $(40 \mathrm{U} / \mu \mathrm{l})$ was added to the worm lysate and centrifuged by $15,000 \times g$ for $15 \mathrm{~min}$ at
$4{ }^{\circ} \mathrm{C}$. For the RNA-cleavage assay $95 \mu \mathrm{l}$ supernatant of the worm lysate with $200 \mu \mathrm{g}$ total protein was incubated with $5 \mathrm{mM} \mathrm{Ca}{ }^{2+}, \mathrm{Mg}^{2+}, \mathrm{Mn}^{2+}$, or $10 \mathrm{mM}$ EGTA for $30 \mathrm{~min}$ at $7{ }^{\circ} \mathrm{C}$ before total RNA was extracted with Trizol. $0.2 \mu \mathrm{g}$ RNA was loaded on 10\% 8 M Urea acrylamide gel with SYBR Gold for visualization of RNA.

Microarray. Both endu-2(tm4977) and endu-2(tm4977);byEx1375[endu-2P::endu2::EGFP;myo-2::mCherry] animals used for microarray were granddaughter decedents of one single endu-2(tm4977);byEx1375[endu-2P::endu-2::EGFP;myo-2:: $m$ Cherry] animals (stain preparation see Supplementary Fig. 8). Animals were raised at $25^{\circ} \mathrm{C}$ for $48 \mathrm{~h}$ after hatching and total RNA was purified by using RNeasy ${ }^{\circledast}$ Mini Kit (Qiagen, Venlo, The Netherlands). The following data analysis were performed with Partek Genomics Suite.

GO term enrichment analysis. GO term and tissue enrichment analysis was carried out with the online enrichment analysis tool (https://wormbase.org/tools/ enrichment/tea/tea.cgi) ${ }^{35}$.

Quantification of mRNA level via quantitative RT-PCR (qPCR). Total RNA was prepared from day-one adult animals raised at 25 or $20^{\circ} \mathrm{C}$ by using RNeasy ${ }^{\circledR}$ Mini Kit (Qiagen, Venlo, The Netherlands). endu-2(tm4977) animals were granddaughter decedents of endu-2(tm4977);byEx1375[endu-2P:::endu-2::EGFP;myo-2:: $m C h e r r y]$ animals. act- 4 was used as internal control. qPCR primer sequences: cav1 forward aagtgctggtggagtagatgc, cav-1 reverse tccgatagcgatgttctctc, clec-169 forward tggacacttgtaactgtgcaaga, clec-169 reverse ttttcatttgaccactttagatcg, F38B6.4 forward cccttgtcctcggaaattaga, F38B6.4 reverse caggcccgaagatggtaat, pfas-1 forward caagattggaagttccgaaga, pfas-1 reverse ctcaatatccggacagtcgtc, B0286.3 forward gacccgaaaacttggagctt, B0286.3 reverse Tggaacgaatgagcacataatc, atic-1 forward tgctacaaaaatgccagcag, atic-1 reverse cgtttaaaggaagaccaacagc, acox-1.2 forward agcggtgatctatggaagtga, acox-1.2 reverse agctcagggatcttggacac, abdh-3.2 forward atgacaccaccccaattgtc, $a b d h-3.2$ reverse tgctgtcatgagtacttcctgtg, col-176 forward ccacaacctttgctccaatc, col-176 reverse gagcacacttgatgcagtcg, clec-15 forward tgcgccagaagggtattact, clec-15 reverse cgcagaacgatctaaccaga, asp-14 forward gctgcagttaccaacattacca, as $p-14$ reverse agcaacgaagaaggttgagg.

Single-molecule fluorescent in situ hybridization (smFISH) and data analysis In total, 38-48 probes of $20 \mathrm{nt}$ in length targeting each mature mRNA were designed with Stellaris RNA FISH probe designer on gene target specificity. Probes were produced, conjugated to CAL Fluor Red 610, and purified by Biocat (sequences of the probe sets are available in Supplementary Data 4). Wild type and endu-2(tm4977) $\mathrm{L} 4$ animals were selected and dissected $24 \mathrm{~h}$ later for smFISH staining using a published protocol procedure ${ }^{36}$. Images were acquired with an Image $\mathrm{Z} 1$ fluorescence microscope. Exposure times and acquisition settings were identical between replicates. mRNA puncta were quantified by using ImageJ $1.51 \mathrm{~s}$ cell counter plug-in. Regions of interest for acquisition were defined by nuclei DAPI staining.

Statistical analysis. Experiments shown in this study were performed independently 2-4 times. Details of the particular statistical analyses used, precise $P$-values, statistical significance, number of biological replicas, and sample sizes for all of the graphs are indicated in the figures or figure legends. $n$ represents the number of animals tested, unless mentioned otherwise. $N$ means the number of biological replicates. ${ }^{* * *} P$-value $<0.0001$.

Reporting summary. Further information on research design is available in the Nature Research Reporting Summary linked to this article.

\section{Data availability}

The authors declare that all data supporting the findings of this study are available within the article and its supplementary information files or from the corresponding author upon reasonable request. New raw data: Microarray data have been deposited in the ArrayExpresss database under accession code E-MTAB-7993. RIP-seq data have been deposited in BioProject under BioProject ID PRJNA544611. RNA-seq data of isolated gonad have been deposited in BioProject under ID PRJNA655512. Published data used in this study: The whole animal RNA-seq data ${ }^{20}$ are downloaded from Gene Expression Ominibus under accession code: GSE101524. The analyzed microarray, RIP-Seq and RNA-seq data of isolated gonad are included in Supplementary Data 1-3.

Received: 28 September 2020; Accepted: 28 January 2021; Published online: 24 February 2021

\section{References}

1. Ahmed, S. \& Hodgkin, J. MRT-2 checkpoint protein is required for germline immortality and telomere replication in C. elegans. Nature 403, 159-164 (2000). 
2. Katz, D. J., Edwards, T. M., Reinke, V. \& Kelly, W. G. A C. elegans LSD1 demethylase contributes to germline immortality by reprogramming epigenetic memory. Cell 137, 308-320 (2009).

3. Lev, I. et al. MET-2-dependent H3K9 methylation suppresses transgenerational small RNA inheritance. Curr. Biol. 27, 1138-1147 (2017).

4. Weiser, N. E. et al. MORC-1 integrates nuclear RNAi and transgenerational chromatin architecture to promote germline immortality. Dev. Cell 41, 408-423 e407 (2017).

5. Buckley, B. A. et al. A nuclear Argonaute promotes multigenerational epigenetic inheritance and germline immortality. Nature 489, 447-451 (2012).

6. Simon, M. et al. Reduced insulin/IGF-1 signaling restores germ cell immortality to Caenorhabditis elegans Piwi mutants. Cell Rep. 7, 762-773 (2014).

7. Barucci, G. et al. Small-RNA-mediated transgenerational silencing of histone genes impairs fertility in piRNA mutants. Nat. Cell Biol. 22, 235-245 (2020).

8. Hackbart, M., Deng, X. \& Baker, S. C. Coronavirus endoribonuclease targets viral polyuridine sequences to evade activating host sensors. Proc. Natl Acad. Sci. USA 117, 8094-8103 (2020).

9. Ivanov, K. A. et al. Major genetic marker of nidoviruses encodes a replicative endoribonuclease. Proc. Natl Acad. Sci. USA 101, 12694-12699 (2004).

10. Ujisawa, T. et al. Endoribonuclease ENDU-2 regulates multiple traits including cold tolerance via cell autonomous and nonautonomous controls in Caenorhabditis elegans. Proc. Natl Acad. Sci. USA 115, 8823-8828 (2018).

11. Jia, F., Chi, C. \& Han, M. Regulation of nucleotide metabolism and germline proliferation in response to nucleotide imbalance and genotoxic stresses by EndoU nuclease. Cell Rep. 30, 1848-1861 e1845 (2020).

12. Kelly, W. G., Xu, S., Montgomery, M. K. \& Fire, A. Distinct requirements for somatic and germline expression of a generally expressed Caernorhabditis elegans gene. Genetics 146, 227-238 (1997).

13. Grant, B. \& Greenwald, I. The Caenorhabditis elegans sel-1 gene, a negative regulator of lin-12 and glp-1, encodes a predicted extracellular protein. Genetics 143, 237-247 (1996).

14. Tijsterman, M., Okihara, K. L., Thijssen, K. \& Plasterk, R. H. PPW-1, a PAZ/ PIWI protein required for efficient germline RNAi, is defective in a natural isolate of C. elegans. Curr. Biol. 12, 1535-1540 (2002).

15. Ni, J. Z. et al. A transgenerational role of the germline nuclear RNAi pathway in repressing heat stress-induced transcriptional activation in C. elegans. Epigenetics Chromatin 9, 3 (2016).

16. Alcazar, R. M., Lin, R. \& Fire, A. Z. Transmission dynamics of heritable silencing induced by double-stranded RNA in Caenorhabditis elegans. Genetics 180, 1275-1288 (2008).

17. Gioia, U. et al. Functional characterization of XendoU, the endoribonuclease involved in small nucleolar RNA biosynthesis. J. Biol. Chem. 280 , 18996-19002 (2005).

18. Schwarz, D. S. \& Blower, M. D. The calcium-dependent ribonuclease XendoU promotes ER network formation through local RNA degradation. J. Cell Biol. 207, 41-57 (2014).

19. Ortiz, M. A., Noble, D., Sorokin, E. P. \& Kimble, J. A new dataset of spermatogenic vs. oogenic transcriptomes in the nematode Caenorhabditis elegans. G3 4, 1765-1772 (2014).

20. Gomez-Orte, E. et al. Effect of the diet type and temperature on the C. elegans transcriptome. Oncotarget 9, 9556-9571 (2018)

21. Sharma, U. et al. Small RNAs are trafficked from the epididymis to developing mammalian sperm. Dev. Cell 46, 481-494 e486 (2018).

22. Maciel, E. \& Mansuy, I. M. Extracellular vesicles and their miRNA Cargo: a means of communication between soma and germline in the mammalian reproductive system. Chim. (Aarau) 73, 356-361 (2019).

23. Muir, V. S., Gasch, A. P. \& Anderson, P. The substrates of nonsense-mediated mRNA decay in Caenorhabditis elegans. G3 8, 195-205 (2018).

24. Knutson, A. K., Egelhofer, T., Rechtsteiner, A. \& Strome, S. Germ granules prevent accumulation of somatic transcripts in the adult Caenorhabditis elegans germline. Genetics 206, 163-178 (2017).

25. Poe, J. C. et al. EndoU is a novel regulator of AICD during peripheral B cell selection. J. Exp. Med. 211, 57-69 (2014).

26. Laneve, P. et al. Drosophila CG3303 is an essential endoribonuclease linked to TDP-43-mediated neurodegeneration. Sci. Rep. 7, 41559 (2017).

27. Kim, Y. et al. Crystal structure of Nsp15 endoribonuclease NendoU from SARS-CoV-2. Protein Sci. https://doi.org/10.1002/pro.3873 (2020).

28. Inaba, N., Renk, T. \& Bohn, H. Immunohistochemical location of placental proteins (PP8, 9, 10, 11, 12) in human term placentae. Arch. Gynecol. 230, 109-121 (1980).

29. Crittenden, S. \& Kimble, J. Preparation and immunolabeling of Caenorhabditis elegans. Cold Spring Harb. Protoc. 2009, https://doi.org/ 10.1101/pdb.prot5216 (2009).
30. Afgan, E. et al. The Galaxy platform for accessible, reproducible and collaborative biomedical analyses: 2018 update. Nucleic Acids Res. 46, W537-W544 (2018)

31. Bolger, A. M., Lohse, M. \& Usadel, B. Trimmomatic: a flexible trimmer for Illumina sequence data. Bioinformatics 30, 2114-2120 (2014).

32. Dobin, A. et al. STAR: ultrafast universal RNA-seq aligner. Bioinformatics 29 , 15-21 (2013).

33. Liao, Y., Smyth, G. K. \& Shi, W. featureCounts: an efficient general purpose program for assigning sequence reads to genomic features. Bioinformatics $\mathbf{3 0}$ 923-930 (2014)

34. Panda, A. C., Martindale, J. L. \& Gorospe, M. Affinity pulldown of biotinylated RNA for detection of protein-RNA complexes. Bio Protoc 6 , https://doi.org/10.21769/BioProtoc.2062 (2016).

35. Angeles-Albores, D., Lee, RaymondY. N., Chan, J. \& Sternberg, P. W. Tissue enrichment analysis for C. elegans genomics. BMC Bioinforma. 17, 366 (2016).

36. Lee, C., Sorensen, E. B., Lynch, T. R. \& Kimble, J. C. elegans GLP-1/Notch activates transcription in a probability gradient across the germline stem cell pool. Elife 5, https://doi.org/10.7554/eLife.18370 (2016).

\section{Acknowledgements}

We thank the Caenorhabditis elegans Genetics Center which is funded by the NIH Office of Research Infrastructure Programs (P40 OD010440) for providing some strains; Dr. S Mitani for providing endu-2(tm4977) strain, Cornelia Habacher for sharing the RIP-Sec protocol, D. Sherwood for providing the fos-1 promoter. We thank the staff of the Life Imaging Center (LIC) of the Albert-Ludwigs-University Freiburg for their microscopy resources. This work was funded by grants from the German Research Foundation (DFG) (SFB850, SFB1381), Germany's Excellence strategy (CIBSS-EXC-2189 - Project ID 8 390939984) and the German Excellence Initiative (BIOSS - EXC294) to R.B.

\section{Author contributions}

W.Q. and R.B. designed the experiments and analyzed data. W.Q., E.D.v.G., F.X., Q.Z., L.L., and W.Y. performed the experiments. D.P. performed and analyzed microarray. W. Q. and W.M. analyzed RNA-Seq data. W.Q. and R.B. wrote the manuscript.

\section{Funding}

Open Access funding enabled and organized by Projekt DEAL.

\section{Competing interests}

The authors declare no competing interests.

\section{Additional information}

Supplementary information The online version contains supplementary material available at https://doi.org/10.1038/s41467-021-21516-6.

Correspondence and requests for materials should be addressed to W.Q.

Peer review information Nature Communications thanks Scott Kennedy and the other anonymous, reviewer(s) for their contribution to the peer review of this work. Peer reviewer reports are available.

Reprints and permission information is available at http://www.nature.com/reprints

Publisher's note Springer Nature remains neutral with regard to jurisdictional claims in published maps and institutional affiliations.

Open Access This article is licensed under a Creative Commons Attribution 4.0 International License, which permits use, sharing, adaptation, distribution and reproduction in any medium or format, as long as you give appropriate credit to the original author(s) and the source, provide a link to the Creative Commons license, and indicate if changes were made. The images or other third party material in this article are included in the article's Creative Commons license, unless indicated otherwise in a credit line to the material. If material is not included in the article's Creative Commons license and your intended use is not permitted by statutory regulation or exceeds the permitted use, you will need to obtain permission directly from the copyright holder. To view a copy of this license, visit http://creativecommons.org/ licenses/by/4.0/.

(c) The Author(s) 2021, corrected publication 2021 